\title{
A Fully Discretized Finite Element Approximation for an Incompressible Flow in Porous Media
}

\author{
Abdellatif Agouzal ${ }^{1}$, Karam Allali ${ }^{2, *}$, Siham Binna $^{3}$ \\ ${ }^{1}$ University Lyon1, CNRS UMR 5208, Institute Camille Jordan, 69100 Villeurbanne, France \\ ${ }^{2}$ University Hassan II, FSTM, PO Box 146, Mohammadia, Morocco
}

Copyright (C) 2015 by authors, all rights reserved. Authors agree that this article remains permanently

open access under the terms of the Creative Commons Attribution License 4.0 International License

\begin{abstract}
In this paper, we will study the fully discretized finite element approximation for an incompressible flow in porous media. The model consists of the heat equation, the equation for the concentration and the equations of motion under the Darcy law. The model is rewritten using the stream function-vorticity formulation. The Stability of the fully discrete problem is established. Optimal a priori error estimates are given.
\end{abstract}

Keywords A Priori Error Estimates, Darcy Law, Finite Element, Porous Media

AMS Subject Classification 35B45, 65N30, 76S05

\section{Introduction}

Within the last decades, a significant number of works has been devoted to the reaction front propagation in porous media (see for instance $[1,2,3]$ and the references therein). The vast majority of works used the velocity-pressure formulation in their study via the Darcy equation. However, the stream function-vorticity formulation is particulary used to perform the numerical simulation of an incompressible fluid flow in porous media [4, 5]. Among the advantages of this formulation is to reduce the unknows of the numerical problem. In this paper, we are interested in studying the finite element of stream function-vorticity formulation for propagating reaction front in porous media. The model considered is a system of reactiondiffusion equations coupled with the hydrodynamic under the Darcy-Boussinesq approximation in the open bounded convex domain $\Omega \subset \mathbb{R}^{2}[6,7,8]$ :

$$
(P)\left\{\begin{array}{l}
\partial_{t} T-\lambda \Delta T+u . \nabla T=\alpha C \exp \left(-\frac{E}{R T}\right), \\
\partial_{t} C-\eta \Delta C+u \cdot \nabla C=-\alpha C \exp \left(-\frac{E}{R T}\right), \\
\partial_{t} u+\frac{\mu}{K} u+\nabla p=\beta\left(T-T_{0}\right) g \gamma \\
\operatorname{div} u=0
\end{array}\right.
$$

where $T$ is the temperature, $C$ is the concentration, $u$ is the velocity, $p$ is the pressure, $\lambda$ is the thermal diffusivity, $\eta$ is the diffusion, $\mu$ is the viscosity, $K$ is the permeability, $E$ is the activation energy, $R$ is the universal gas constant, $\alpha$ is the Arrhenius pre-exponential factor, $T_{0}$ is a mean value of temperature, $g$ is the gravity, $\gamma$ is the upward unit vector and $\beta$ is the coefficient of the thermal expansion of the fluid.

The boundary conditions are of Dirichlet-Neumann type for the temperature, the concentration and of impermeability 
type for the normal component of velocity:

$$
\left\{\begin{array}{l}
\left.T\right|_{\Gamma_{1}}=\left.C\right|_{\Gamma_{1}}=0,\left.\quad \frac{\partial T}{\partial n}\right|_{\Gamma_{2}}=\left.\frac{\partial C}{\partial n}\right|_{\Gamma_{2}}=0 \text { and }\left.u \cdot n\right|_{\partial \Omega}=0 \\
\left.u\right|_{t=0}=u_{0},\left.\quad T\right|_{t=0}=T_{0} \text { and }\left.C\right|_{t=0}=C_{0}
\end{array}\right.
$$

where $\Gamma_{1}$ and $\Gamma_{2}$ are disjoined opens parts of $\partial \Omega$ such that $\overline{\Gamma_{1}} \cup \overline{\Gamma_{2}}=\partial \Omega$.

Due to the incompressibility of the fluid, we introduce the stream function $\psi$ defined by: $u=\operatorname{rot}(\psi)$, by introducing also the vorticity $\omega=\operatorname{curl}(u)$, the problem $(\mathcal{P})$ becomes:

$$
(\mathcal{P}) \quad\left\{\begin{array}{l}
\partial_{t} T-\lambda \Delta T+\operatorname{rot}(\psi) \cdot \nabla T-C g(T)=0 \\
\partial_{t} C-\eta \Delta C+\operatorname{rot}(\psi) \cdot \nabla C+C g(T)=0 \\
\partial_{t} \omega+\mu_{p} \omega=f(T), \\
-\Delta \psi=\omega
\end{array}\right.
$$

where $g(T)=\alpha \exp \left(-\frac{E}{R T}\right), f(T)=\beta g \frac{\partial T}{\partial x}$, and $\mu_{p}=\frac{\mu}{K}$.

Because of there is no flow of fluid through the boundary (impermeability boundary), we will have the following zero-flux condition:

$$
\left.\psi\right|_{\partial \Omega}=0
$$

The existence and uniqueness of solutions has been studied in [9], while the finite elements approximations of the semidiscretized problem has been established in [10]; the authors studied the existence and the uniqueness of the semi-discrete solution and give some error estimates on the problem unknowns. In this work, attention is focused to the fully discretized problem. First, we will study the stability of the numerical scheme. Next, we will give some optimal error estimates on vorticity, stream function, concentration, pressure and temperature.

\section{The numerical stability of the problem}

\subsection{The variational formulation of the problem}

Before giving the problem in its variational form; first, we will describe the functional framework. We set

$$
X=L^{2}\left(0, t, H_{0}^{1}(\Omega)\right), \quad W=L^{2}\left(0, t, H_{0, \Gamma_{1}}^{1}(\Omega)\right) \text { and } M=L^{2}\left(0, t, L^{2}(\Omega)\right) .
$$

The variational form of the continued $\operatorname{problem}(\mathcal{P})$ can be written in the following form:

$$
\left(\mathcal{P}_{v}\right)\left\{\begin{array}{l}
\text { Find a }(\psi, \omega, T, C) \in X \times \bar{M} \times \bar{W}^{2}, \text { such as, for all }(\zeta, v, \phi, \xi) \in X \times M \times W^{2}, \text { we have : } \\
\left(\partial_{t} T, \phi\right)+\lambda(\nabla T, \nabla \phi)+\frac{1}{2} \int_{\Omega}(\operatorname{rot}(\psi) \nabla T) \phi-\frac{1}{2} \int_{\Omega}(\operatorname{rot}(\psi) \nabla \phi) T=\int_{\Omega} C g(T) \phi \\
\left(\partial_{t} C, \xi\right)+\eta(\nabla C, \nabla \xi)+\frac{1}{2} \int_{\Omega}(\operatorname{rot}(\psi) \nabla C) \xi-\frac{1}{2} \int_{\Omega}(\operatorname{rot}(\psi) \nabla \xi) C=-\int_{\Omega} C g(T) \xi \\
\left(\partial_{t} \omega, v\right)+\mu_{p}(\omega, v)=\int_{\Omega} f(T) v \\
(\nabla \psi, \nabla \zeta)=(\omega, \zeta),
\end{array}\right.
$$

where

$$
\bar{M}=\left\{\omega \in M, \quad \frac{\partial \omega}{\partial t} \in L^{2}\left(0, t,\left(L^{2}(\Omega)\right)\right\} \text { and } \bar{W}=\left\{T \in W, \frac{\partial T}{\partial t} \in L^{2}\left(0, t, H_{0, \Gamma_{1}}^{1}(\Omega)\right)^{*}\right\} .\right.
$$

The existence of the weak solution of problem $\left(\mathcal{P}_{v}^{n}\right)$ has been established in Reference [9]. We can see clearly that the 
parameters and the functions $f$ and $g$ of the problem verify the following conditions:

$$
\left\{\begin{array}{l}
\text { The reals } \mu_{p}, \quad \eta \text { and } \lambda \text { are strictly positives, } \\
g \in W^{1, \infty}(\mathbb{R}), \quad\left\|g^{\prime}\right\|_{L^{\infty}(\Omega)}=\frac{E \alpha}{R T_{i}^{2}}, g \geq 0 \text { and }\|g\|_{L^{\infty}(\Omega)}=\alpha, \\
f \in W^{1, \infty}(\mathbb{R}), \quad f\left(T_{0}\right)=0 \text { and } \\
\forall\left(T_{1}, T_{2}\right) \in\left(H_{0, \Gamma_{1}}^{1}(\Omega)\right)^{2}, \quad\left\|f\left(T_{1}\right)-f\left(T_{2}\right)\right\|_{L^{2}(\Omega)} \leq \beta g\left\|\nabla\left(T_{1}-T_{2}\right)\right\|_{L^{2}(\Omega)},
\end{array}\right.
$$

where $T_{i}$ is the temperature of the unburned mixture. We introduce now the constant of Friedrichs-Poincaré which depends on the geometry of the domain $\Omega$ :

$$
\rho=\sup _{u \in H_{0}^{1}(\Omega)} \frac{\|u\|_{L^{2}(\Omega)}}{\|\nabla u\|_{L^{2}(\Omega)}} .
$$

\subsection{The fully semi-discrete problem}

In order to give the semi-discrete problem, we will need the following spaces:

$$
X_{h} \subset H_{0}^{1}(\Omega), \quad M_{h} \subset L^{2}(\Omega) \text { and } W_{h} \subset H_{0, \Gamma_{1}}^{1}(\Omega),
$$

where $h$ is a strictly positive constant. We assume that the spaces $X_{h}, M_{h}$ and $W_{h}$ satisfies the following assumptions:

1. For all $0<\sigma \leq 1$, there exist a linear continuous operator $R_{h}$ from $W^{1+\sigma, 4}(\Omega) \cap \stackrel{\circ}{W^{1,4}}(\Omega)$ onto $X_{h}$, such that: for all $\psi \in W^{1+\sigma, 4}(\Omega) \cap \stackrel{\circ}{W}^{1,4}(\Omega)$,

$$
\left\|\psi-R_{h} \psi\right\|_{1,4} \lesssim h^{\sigma}|\psi|_{1+\sigma, 4}
$$

and

$$
\int_{\Omega} \nabla\left(\psi-R_{h} \psi\right) \nabla v_{h}=0 \quad \forall v_{h} \in X_{h}
$$

2. For all $0<\sigma \leq 1$, there exist a linear continuous operator $i_{h}$ from $H^{1+\sigma}(\Omega) \cap H_{0}^{1}(\Omega)$ onto $W_{h}$ such that: for all $T \in H^{1+\sigma}(\Omega) \cap H_{0}^{1}(\Omega)$,

and

$$
\left\|T-i_{h} T\right\|_{1, \Omega} \lesssim h^{\sigma}|T|_{1+\sigma, \Omega}
$$

$$
\int_{\Omega} \nabla\left(T-i_{h} T\right) \nabla v_{h}=0, \quad \forall v_{h} \in X_{h} .
$$

Examples of such spaces verifying these conditions is given in [11] and [12]. For convenience, for all $(T, \phi, C, \psi) \in W^{4}$, we introduce the forms defined by:

$$
\left\{\begin{array}{l}
a_{1}(u, T, \phi)=\frac{1}{2}\left(\int_{\Omega}(\operatorname{rot}(u) \nabla) T \phi d x-\int_{\Omega}(\operatorname{rot}(u) \nabla) \phi T d x\right), \\
j(T, \psi)=\int_{\Omega} \nabla T \nabla \psi d x \\
Z(C, T, \psi)=\int_{\Omega} C g(T) \psi d x .
\end{array}\right.
$$

The fully semi-discretized problem can be writhen as follows:

Let $k=\Delta t$ be a small parameter, the fully discrete problem is given by: For $C_{h}^{n}, T_{h}^{n}, \psi_{h}^{n}, \omega_{h}^{n}$ known, find $C_{h}^{n+1} \in W_{h}, T_{h}^{n+1} \in W_{h}, \psi_{h}^{n+1} \in X_{h}$ and $\omega_{h}^{n+1} \in M_{h}$ such that:

$$
\left(\mathcal{P}_{h}^{n}\right) \quad\left\{\begin{array}{c}
\forall \phi_{h} \in W_{h}, \begin{array}{r}
\left(C_{h}^{n+1}, \phi_{h}\right)_{0, \Omega}+k \eta j\left(C_{h}^{n+1}, \phi_{h}\right)+k a_{1}\left(\psi_{h}^{n}, C_{h}^{n+1}, \phi_{h}\right) \\
\quad \\
\quad k \mathrm{Z}\left(C_{h}^{n+1}, T_{h}^{n}, \phi_{h}\right)=\left(C_{h}^{n}, \phi_{h}\right)_{0, \Omega},
\end{array} \\
\forall \phi_{h} \in W_{h},\left(T_{h}^{n+1}, \phi_{h}\right)_{0, \Omega}+k \lambda j\left(T_{h}^{n+1}, \phi_{h}\right)+k a_{1}\left(\psi_{h}^{n}, T_{h}^{n+1}, \phi_{h}\right) \\
\quad-k \mathrm{Z}\left(C_{h}^{n+1}, T_{h}^{n}, \phi_{h}\right)=\left(T_{h}^{n}, \phi_{h}\right)_{0, \Omega}, \\
\forall v_{h} \in M_{h}, \quad \begin{array}{r}
\left(\omega_{h}^{n+1}, v_{h}\right)_{0, \Omega}+k \mu_{p}\left(\omega_{h}^{n+1}, v_{h}\right)_{0, \Omega}-k \\
=k\left(f\left(T_{h}^{n+1}\right), v_{h}\right)_{0, \Omega}+\left(\omega_{h}^{n}, v_{h}\right)_{0, \Omega}
\end{array} \\
\forall q_{h} \in X_{h}, j\left(\psi_{h}^{n+1}, q_{h}\right)=\left(\omega_{h}^{n+1}, q_{h}\right)_{0, \Omega} .
\end{array}\right.
$$

By classical arguments $[11,13]$, we have the following lemma:

Lemma 2.1. For all $n \in N^{*}$, the problem admits a unique solution

$$
\left(\psi_{h}^{n}, \omega_{h}^{n}, T_{h}^{n}, C_{h}^{n}\right) \in X_{h} \times M_{h} \times W_{h}^{2} .
$$


In the sequel of this paper, for each $n$ positive integer, we denoted by $C_{h}^{n}, T_{h}^{n}, \psi_{h}^{n}$ and $\omega_{h}^{n}$ the discrete solution of the problem $\left(\mathcal{P}_{h}^{n}\right)$.

In the following, we set:

$$
\left\{\begin{array}{l}
N_{\psi}=\|\psi\|_{L^{\infty}\left(0, t, W^{1,4}(\Omega)\right)}, \quad M_{\psi}=\sup _{0 \leq k \leq N}\left\|\psi_{h}^{k}\right\|_{1,4} \\
N_{T}=\|T\|_{L^{\infty}\left(0, t, H^{1}(\Omega)\right)}, \quad N_{C}=\|C\|_{L^{\infty}\left(0, t, H^{1}(\Omega)\right)} \\
\bar{\partial}_{t} \phi^{n}=\frac{\phi^{n}-\phi^{n-1}}{k}
\end{array}\right.
$$

The main theorem of the paper is the following:

Theorem 2.1. Assume that the following conditions are verified: $k \leq \frac{1}{4}$ and

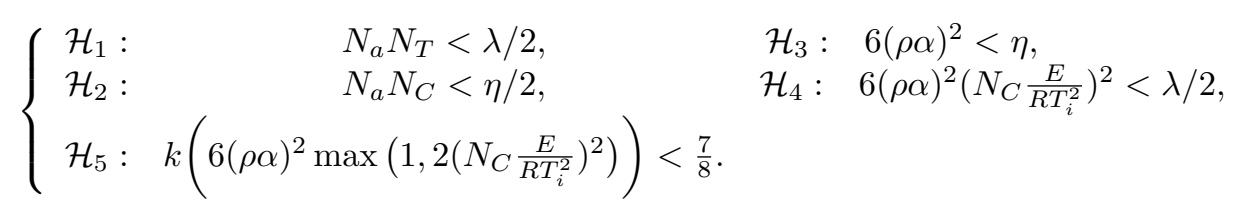

If the solution of the problem $\left(\mathcal{P}_{h}^{n}\right)$ admits the following regularity:

$$
\left\{\begin{array}{l}
\omega \in W^{2, \infty}\left(0, t, L^{2}(\Omega)\right) \cap W^{1, \infty}\left(0, t, L^{2}(\Omega)\right) \cap L^{\infty}\left(0, t, H^{\sigma}(\Omega)\right) \\
\psi \in L^{\infty}\left(0, t, W^{1+\sigma, 4}(\Omega)\right), \\
T, C \in W^{2, \infty}\left(0, t, L^{2}(\Omega)\right) \cap W^{1, \infty}\left(0, t, H^{1}(\Omega)\right) \cap L^{\infty}\left(0, t, H^{1+\sigma}(\Omega)\right),
\end{array}\right.
$$

Then, we have the following error estimates:

$$
\left\|\psi^{n}-\psi_{h}^{n}\right\|_{1,4}^{2} \lesssim\left(h^{2 \sigma}+k^{2}\right)\left(k n+k^{2} n^{2}\right)
$$

for the stream function,

$$
\left(1+\frac{k \mu_{p}}{2}\right)\left\|\omega^{n}-\omega_{h}^{n}\right\|_{L^{2}(\Omega)}^{2} \lesssim\left(h^{2 \sigma}+k^{2}\right)\left(k n+k^{2} n^{2}+k^{3} n^{3}+k^{4} n^{4}\right)
$$

for the vorticity,

$$
\left\|T^{n}-T_{h}^{n}\right\|_{0, \Omega}^{2}+\frac{8}{7(1-w)} k \min \left(\lambda, \eta-6(\alpha \rho)^{2}\right)\left\|\nabla\left(T^{n}-T_{h}^{n}\right)\right\|_{0, \Omega}^{2} \lesssim\left(h^{2 \sigma}+k^{2}\right)\left(k n+k^{2} n^{2}+k^{3} n^{3}\right)
$$

for the temperature and

$$
\left\|C^{n}-C_{h}^{n}\right\|_{0, \Omega}^{2}+\frac{8}{7(1-w)} k \min \left(\lambda, \eta-6(\alpha \rho)^{2}\right)\left\|\nabla\left(C^{n}-C_{h}^{n}\right)\right\|_{0, \Omega}^{2} \lesssim\left(h^{2 \sigma}+k^{2}\right)\left(k n+k^{2} n^{2}+k^{3} n^{3}\right)
$$

for the concentration.

Here $0<\sigma \leq 1$ and $w=\frac{8 k}{7}(6 \rho \alpha)^{2} \max \left(1,2\left(N_{C} \frac{E}{R T_{b}^{2}}\right)^{2}\right)$.

\subsection{Study of stability}

The main result of this subsection is to prove stability of our fully-discrete approximate scheme. For this we need some technical lemmas. First we have

Lemma 2.2. For all $n \in N^{*}$, we have

$$
\left\|C_{h}^{n}\right\|_{0, \Omega}^{2}+\sum_{i=0}^{n-1}\left\|C_{h}^{i+1}-C_{h}^{i}\right\|_{0, \Omega}^{2}+2 k \eta \sum_{i=1}^{n}\left\|C_{h}^{i}\right\|_{1, \Omega}^{2} \leq\left\|C_{h}^{0}\right\|_{0, \Omega}^{2} .
$$

Proof. First of all, let us notice that

$$
a_{1}\left(u_{h}^{n}, C_{h}^{n+1}, C_{h}^{n+1}\right)=0 \quad \text { and } \quad \mathrm{z}\left(C_{h}^{n+1}, T_{h}^{n}, C_{h}^{n+1}\right) \geq 0 .
$$

By choosing $C_{h}^{n+1}$ as test function in the first equation of the problem $\left(\mathcal{P}_{h}^{n}\right)$ and using 3.1, it follows

$$
\left\|C_{h}^{n+1}\right\|_{0, \Omega}^{2}+\left\|C_{h}^{n+1}-C_{h}^{n}\right\|_{0, \Omega}^{2}+2 k \eta\left\|C_{h}^{n+1}\right\|_{1, \Omega}^{2} \leq\left\|C_{h}^{n}\right\|_{0, \Omega}^{2}
$$

Now, by summing over time, we obtain

$$
\left\|C_{h}^{n+1}\right\|_{0, \Omega}^{2}+\sum_{i=0}^{n}\left\|C_{h}^{i+1}-C_{h}^{i}\right\|_{0, \Omega}^{2}+2 k \eta \sum_{i=1}^{n+1}\left\|C_{h}^{i}\right\|_{1, \Omega}^{2} \leq\left\|C_{h}^{0}\right\|_{0, \Omega}^{2} .
$$


Lemma 2.3. For all $n \in N^{*}$, we have

$$
\left\|T_{h}^{n}\right\|_{0, \Omega}^{2}+\sum_{i=0}^{n-1}\left\|T_{h}^{i+1}-T_{h}^{i}\right\|_{0, \Omega}^{2}+\lambda k \sum_{i=1}^{n}\left\|T_{h}^{i}\right\|_{1, \Omega}^{2} \leq\left\|T_{h}^{0}\right\|_{0, \Omega}^{2}+\frac{\alpha^{2} \rho^{4}}{2 \lambda \eta}\left\|C_{h}^{0}\right\|_{0, \Omega}
$$

Proof. By choosing $T_{h}^{n+1}$ as test function in the second equation of the problem $\left(\mathcal{P}_{h}^{n}\right)$ and using (3.1) we have

$$
\left\|T_{h}^{n+1}\right\|_{0, \Omega}^{2}+\left\|T_{h}^{n+1}-T_{h}^{n}\right\|_{0, \Omega}^{2}+2 k \lambda\left\|T_{h}^{n+1}\right\|_{1, \Omega}^{2} \leq 2 k \mathrm{Z}\left(C_{h}^{n+1}, T_{h}^{n}, T_{h}^{n+1}\right)+\left\|T_{h}^{n}\right\|_{0, \Omega}^{2} .
$$

However

$$
\mathrm{Z}\left(C_{h}^{n+1}, T_{h}^{n}, T_{h}^{n+1}\right) \leq \frac{\alpha^{2} \rho^{4}}{2 \lambda}\left\|C_{h}^{n+1}\right\|_{1, \Omega}^{2}+\frac{\lambda}{2}\left\|T_{h}^{n+1}\right\|_{1, \Omega}^{2}
$$

Therefore

$$
\left\|T_{h}^{n+1}\right\|_{0, \Omega}^{2}+\left\|T_{h}^{n+1}-T_{h}^{n}\right\|_{0, \Omega}^{2}+k \lambda\left\|T_{h}^{n+1}\right\|_{1, \Omega}^{2} \leq\left\|T_{h}^{n}\right\|_{0, \Omega}^{2}+k \frac{\alpha^{2} \rho^{4}}{\lambda} \|\left(C_{h}^{n+1} \|_{1, \Omega}^{2} .\right.
$$

By summing over time and using Lemma 2.2, We conclude

$$
\left\|T_{h}^{n+1}\right\|_{0, \Omega}^{2}+\sum_{i=0}^{n}\left\|T_{h}^{i+1}-T_{h}^{i}\right\|_{0, \Omega}^{2}+k \lambda \sum_{i=1}^{n+1}\left\|T_{h}^{i}\right\|_{1, \Omega}^{2} \leq\left\|T_{h}^{0}\right\|_{0, \Omega}^{2}+\frac{\alpha^{2} \rho^{4}}{2 \eta \lambda}\left\|C_{h}^{0}\right\|_{1, \Omega}^{2} .
$$

First we have

Lemma 2.4. For any local solution $\omega_{h}^{n}$ of the problem $\left(\mathcal{P}_{h}^{n}\right)$, we have the estimate:

$$
\left\|\omega_{h}^{n+1}\right\|_{0, \Omega}^{2}+\sum_{i=0}^{n}\left\|\omega_{h}^{i+1}-\omega_{h}^{i}\right\|_{0, \Omega}^{2}+\frac{k \mu_{p}}{2} \sum_{i=1}^{n+1}\left\|\omega_{h}^{i}\right\|_{0, \Omega}^{2} \leq \frac{1}{2 \mu_{p} \lambda}\left(\left\|T_{h}^{0}\right\|_{0, \Omega}^{2}+\frac{\alpha^{2} \rho^{4}}{2 \lambda \eta}\left\|C_{h}^{0}\right\|_{0, \Omega}\right)+\left\|\omega_{h}^{0}\right\|_{L^{2}(\Omega)}^{2}
$$

Proof. By choosing $v_{h}=\omega_{h}^{n}$, as test function in the first equation of the problem $\left(\mathcal{P}_{h}^{n}\right)$, we have:

$$
\left\|\omega_{h}^{n+1}\right\|_{0, \Omega}^{2}+\left\|\omega_{h}^{n+1}-\omega_{h}^{n}\right\|_{0, \Omega}^{2}+k \mu_{p}\left\|\omega_{h}^{n+1}\right\|_{L^{2}(\Omega)}^{2} \leq k\left\|\nabla T_{h}^{n+1}\right\|_{L^{2}(\Omega)}\left\|\omega_{h}^{n+1}\right\|_{L^{2}(\Omega)}+\left\|\omega_{h}^{n}\right\|_{0, \Omega}^{2} .
$$

By using the triangular inequality, we get:

$$
\left\|\omega_{h}^{n+1}\right\|_{0, \Omega}^{2}+\left\|\omega_{h}^{n+1}-\omega_{h}^{n}\right\|_{0, \Omega}^{2}+\frac{k \mu_{p}}{2}\left\|\omega_{h}^{n+1}\right\|_{L^{2}(\Omega)}^{2} \leq \frac{k}{2 \mu_{p}}\left\|\nabla T_{h}^{n+1}\right\|_{L^{2}(\Omega)}^{2}+\left\|\omega_{h}^{n}\right\|_{0, \Omega}^{2} .
$$

Now, by summing over time, we obtain

$$
\left\|\omega_{h}^{n+1}\right\|_{0, \Omega}^{2}+\sum_{i=0}^{n}\left\|\omega_{h}^{i+1}-\omega_{h}^{i}\right\|_{0, \Omega}^{2}+\frac{k \mu_{p}}{2} \sum_{i=1}^{n+1}\left\|\omega_{h}^{i}\right\|_{0, \Omega}^{2} \leq \frac{k}{2 \mu_{p}} \sum_{i=1}^{n+1}\left\|\nabla T_{h}^{i}\right\|_{L^{2}(\Omega)}^{2}+\left\|\omega_{h}^{0}\right\|_{L^{2}(\Omega)}^{2} .
$$

However, using lemma 2.3, get:

$$
\left\|\omega_{h}^{n+1}\right\|_{0, \Omega}^{2}+\sum_{i=0}^{n}\left\|\omega_{h}^{i+1}-\omega_{h}^{i}\right\|_{0, \Omega}^{2}+\frac{k \mu_{p}}{2} \sum_{i=1}^{n+1}\left\|\omega_{h}^{i}\right\|_{0, \Omega}^{2} \leq \frac{1}{2 \mu_{p} \lambda}\left(\left\|T_{h}^{0}\right\|_{0, \Omega}^{2}+\frac{\alpha^{2} \rho^{4}}{2 \lambda \eta}\left\|C_{h}^{0}\right\|_{0, \Omega}\right)+\left\|\omega_{h}^{0}\right\|_{L^{2}(\Omega)}^{2} .
$$

Finally, for the velocity, the following result holds:

Lemma 2.5. For any local solution $\psi_{h}^{n}$ of the problem $\left(\mathcal{P}_{h}^{n}\right)$, we have the estimate:

$$
\left|\psi_{h}^{n}\right|_{W^{1,4}(\Omega)}^{2} \lesssim \frac{1}{2 \mu_{p} \lambda}\left(\left\|T_{h}^{0}\right\|_{0, \Omega}^{2}+\frac{\alpha^{2} \rho^{4}}{2 \lambda \eta}\left\|C_{h}^{0}\right\|_{0, \Omega}\right)+\left\|\omega_{h}^{0}\right\|_{L^{2}(\Omega)}^{2} .
$$

Proof. Let $\psi_{h}^{n}$ be the solution of the problem $\left(\mathcal{P}_{h}^{n}\right)$. From $[14,15]$ we have,

$$
\left|\psi_{h}^{n}\right|_{W^{1,4}(\Omega)} \lesssim \sup _{v \in \stackrel{\circ}{W}^{1,4}(\Omega)} \frac{\left\langle\nabla\left(\psi_{h}^{n}\right), \nabla v\right\rangle}{|v|_{W^{1, \frac{4}{3}}(\Omega)}}
$$

However, for all $v \in \stackrel{\circ}{W}^{1,4}(\Omega)$ we have:

$$
<\nabla\left(\psi_{h}^{n}\right), \nabla v>=<\nabla\left(\psi_{h}^{n}\right), \nabla\left(\Pi_{h} v\right)>
$$


where $\Pi_{h}$ is the projection operator defined from $W^{1, \frac{4}{3}}(\Omega)$ onto $X_{h}$, such as,

$$
\left\langle\nabla \phi_{h}, \nabla\left(\Pi_{h} v\right)-\nabla v\right\rangle=0, \quad \forall \phi_{h} \in X_{h}
$$

By choosing $v_{h}=\Pi_{h} v$ as test function in the last equation of the problem $\left(\mathcal{P}_{h}^{n}\right)$, we get:

$$
\left\langle\nabla \psi_{h}^{n}, \nabla \Pi_{h} v\right\rangle=\left\langle\omega_{h}^{n}, \Pi_{h} v\right\rangle .
$$

Therefore

$$
\left\langle\nabla \psi_{h}^{n}, \nabla v\right\rangle \leq\left\|\omega_{h}^{n}\right\|_{L^{2}(\Omega)}\left\|\Pi_{h} v\right\|_{L^{2}(\Omega)} .
$$

Due to the embedding of $W^{1, \frac{4}{3}}(\Omega)$ onto $L^{2}(\Omega)$, we have:

$$
\left\|\Pi_{h} v\right\|_{L^{2}(\Omega)} \lesssim\left|\Pi_{h} v\right|_{W^{1, \frac{4}{3}}(\Omega)}
$$

and due to the propriety of the operator $\Pi_{h}$, we have:

$$
\left|\Pi_{h} v\right|_{W^{1, \frac{4}{3}(\Omega)}} \lesssim|v|_{W^{1, \frac{4}{3}}(\Omega)},
$$

so

$$
\sup _{v \in \dot{O}^{1,4}(\Omega)} \frac{\left\langle\nabla \psi_{h}^{n}, \nabla v\right\rangle}{|v|_{W^{1, \frac{4}{3}}(\Omega)}} \lesssim\left\|\omega_{h}^{n}\right\|_{L^{2}(\Omega)}
$$

We conclude

$$
\left|\psi_{h}^{n}\right|_{W^{1,4}(\Omega)} \lesssim\left\|\omega_{h}^{n}\right\|_{L^{2}(\Omega)} .
$$

However, using lemma 2.4, we have:

$$
\left|\psi_{h}^{n}\right|_{W^{1,4}(\Omega)}^{2} \lesssim \frac{1}{2 \mu_{p} \lambda}\left(\left\|T_{h}^{0}\right\|_{0, \Omega}^{2}+\frac{\alpha^{2} \rho^{4}}{2 \lambda \eta}\left\|C_{h}^{0}\right\|_{0, \Omega}\right)+\left\|\omega_{h}^{0}\right\|_{L^{2}(\Omega)}^{2} .
$$

Now, using the Lemmas 2.2, 2.3, 2.4 and 2.5, we easily deduce the following stability result:

Theorem 2.2. For all $n$ positive integer, the fully-discrete problem $\left(\mathcal{P}_{h}^{n}\right)$ is stable.

\section{The error estimates}

In this subsection, we will prove some error estimates on speed, on the pressure, on the temperature and on the concentration and we will use the following identity

$$
a(a-b)=\frac{1}{2}\left(a^{2}-b^{2}+(a-b)^{2}\right)
$$

First, we have the following technical estimate:

Lemma 3.1. For any $\omega^{n}$ and $\omega_{h}^{n}$ solutions of the problems $\left(\mathcal{P}_{h}^{n}\right)$ and $\left(\mathcal{P}_{h}^{n}\right)$ respectively, we have:

$$
\left\|\omega^{n}-\omega_{h}^{n}\right\|_{L^{2}(\Omega)}^{2}+\frac{k \mu_{p}}{2} \sum_{i=1}^{n}\left\|\omega^{i}-\omega_{h}^{i}\right\|_{L^{2}(\Omega)} \lesssim k n h^{2 \sigma}+\frac{3 k(\beta g)^{2}}{2 \mu_{p}} \sum_{i=1}^{n}\left\|T^{i}-T_{h}^{i}\right\|_{H^{1}(\Omega)}^{2} .
$$

Proof. For $\omega^{n}$ and $\omega_{h}^{n}$ respectively, solutions of the continuous and the semi-discrete problem, we have:

$$
\left(\partial_{t} \omega^{n}-\bar{\partial}_{t} \omega_{h}^{n}, v_{h}\right)_{\kappa}+\mu_{p}\left(\omega^{n}-\omega_{h}^{n}, v_{h}\right)_{\kappa}=\left(f\left(T^{n}\right)-f\left(T_{h}^{n}\right), v_{h}\right)_{\kappa}, \quad \forall v_{h} \in X_{h},
$$

where $\kappa$ is any element of a regular family of affine meshes $\tau_{h}$. Let $\bar{\omega}^{n} h$ be the approximation of $\omega^{n}$ in $X_{h}$ defined by:

$$
\left.{\overline{\omega^{n}}}_{h}\right|_{\kappa}=\frac{1}{\operatorname{mes}(\kappa)} \int_{\kappa} \omega^{n} d x \text {. }
$$

Hence, for all $0<\sigma<1$ and $\omega^{n} \in H^{\sigma}(\Omega)$ we have the following result:

$$
\left\|\omega^{n}-\bar{\omega}^{n} h\right\|_{L^{2}(\kappa)} \lesssim h_{\kappa}^{\sigma}\left\|\omega^{n}\right\|_{H^{\sigma}(\kappa)}
$$


where $h_{\kappa}$ is the diameter of the element $\kappa$. By using the equality (3.2) and setting $\chi_{h}^{n}=\bar{\omega}_{h}{ }_{h}-\omega_{h}^{n}$, we obtain:

$$
\begin{aligned}
\left(\bar{\partial}_{t} \bar{\omega}_{h}-\bar{\partial}_{t} \omega_{h}^{n}, \chi_{h}^{n}\right)_{\kappa}+\mu_{p}\left(\bar{\omega}_{h}-\omega_{h}^{n}, \bar{\omega}_{h}-\omega_{h}^{n}\right)_{\kappa} & \\
& =-\left(\partial_{t} \omega^{n}-\bar{\partial}_{t} \bar{\omega}^{n} h, \bar{\omega}_{h}-\omega_{h}^{n}\right)_{\kappa}+\mu_{p}\left(\bar{\omega}_{h}{ }_{h}-\omega^{n}, \bar{\omega}_{h}-\omega_{h}^{n}\right)_{\kappa} \\
& +\left(f\left(T^{n}\right)-f\left(T_{h}^{n}\right), \bar{\omega}_{h}-\omega_{h}^{n}\right)_{\kappa} .
\end{aligned}
$$

Therefore, we have:

$$
\begin{aligned}
\left(\bar{\partial}_{t} \chi_{h}^{n}, \chi_{h}^{n}\right)_{\kappa}+\mu_{p}\left\|\bar{\omega}_{h}-\omega_{h}^{n}\right\|_{L^{2}(\kappa)}^{2} & \\
\leq & \left(\left\|\partial_{t} \omega^{n}-\bar{\partial}_{t} \bar{\omega}_{h}\right\|_{L^{2}(\kappa)}+\beta g\left\|\nabla\left(T^{n}-T_{h}^{n}\right)\right\|_{L^{2}(\kappa)}\right) \\
& \times\left\|\bar{\omega}_{h}-\omega_{h}^{n}\right\|_{L^{2}(\kappa)}+\mu_{p}\left\|\omega^{n}-\bar{\omega}_{h}\right\|_{L^{2}(\kappa)}\left\|\bar{\omega}_{h}-\omega_{h}^{n}\right\|_{L^{2}(\kappa)} .
\end{aligned}
$$

Then

$$
\begin{aligned}
\frac{1}{2 k}\left(\left\|\chi_{h}^{n}\right\|_{0, \kappa}^{2}-\left\|\chi_{h}^{n-1}\right\|_{0, \kappa}^{2}\right. & \left.+\left\|\chi_{h}^{n}-\chi_{h}^{n-1}\right\|_{0, \kappa}^{2}\right)+\mu_{p}\left\|\bar{\omega}_{h}-\omega_{h}^{n}\right\|_{L^{2}(\kappa)}^{2} \\
& \leq\left(\left\|\partial_{t} \omega^{n}-\bar{\partial}_{t} \omega_{h}\right\|_{L^{2}(\kappa)}+\beta g\left\|\nabla\left(T^{n}-T_{h}^{n}\right)\right\|_{L^{2}(\kappa)}\right)\left\|\overline{\omega^{n}} h-\omega_{h}^{n}\right\|_{L^{2}(\kappa)} \\
& +\mu_{p}\left\|\omega^{n}-\bar{\omega}_{h}\right\|_{L^{2}(\kappa)}\left\|\bar{\omega}_{h}-\omega_{h}^{n}\right\|_{L^{2}(\kappa)} .
\end{aligned}
$$

However, by using the equality (3.1), we can write:

$$
\begin{aligned}
\left\|\chi_{h}^{n}\right\|_{0, \kappa}^{2}+\mu_{p} k\left\|\chi_{h}^{n}\right\|_{0, \kappa}^{2} & \leq \frac{3 k}{2 \mu_{p}}\left\|\partial_{t} \omega^{n}-\bar{\partial}_{t} \omega_{h}^{n}\right\|_{L^{2}(\kappa)}^{2}+\frac{3 k(\beta g)^{2}}{2 \mu_{p}}\left\|\nabla\left(T^{n}-T_{h}^{n}\right)\right\|_{L^{2}(\kappa)}^{2} \\
& +\frac{3 k \mu_{p}}{2}\left\|\bar{\omega}_{h}-\omega^{n}\right\|_{L^{2}(\kappa)}^{2}+\frac{k \mu_{p}}{2}\left\|\overline{\omega^{n}} h-\omega_{h}^{n}\right\|_{L^{2}(\kappa)}^{2}+\left\|\chi_{h}^{n-1}\right\|_{0, \kappa}^{2} .
\end{aligned}
$$

However, for all $0<\sigma \leq 1$ and $\omega^{n} \in H^{1}\left(0, T, H^{\sigma}(\kappa)\right)$, we have

$$
\left\|\partial_{t} \omega^{n}-\bar{\partial}_{t} \omega^{n}{ }_{h}\right\|_{L^{2}(\kappa)}^{2} \lesssim h_{\kappa}^{2 \sigma}\left\|\partial_{t} \omega^{n}\right\|_{H^{\sigma}(\kappa)}^{2} .
$$

By using (3.4) and summing over time, we get:

$$
\begin{aligned}
\left\|\chi_{h}^{n}\right\|_{0, \kappa}^{2}+\frac{k \mu_{p}}{2} \sum_{i=1}^{n}\left\|\chi_{h}^{i}\right\|_{0, \kappa}^{2} & \lesssim k n h_{\kappa}^{2 \sigma}\left(\left\|\omega^{n}\right\|_{H^{\sigma}(\kappa)}+\left\|\partial_{t} \omega^{n}\right\|_{H^{\sigma}(\kappa)}\right)^{2} \\
& +\frac{3 k(\beta g)^{2}}{2 \mu_{p}} \sum_{i=1}^{n}\left\|T^{i}-T_{h}^{i}\right\|_{H^{1}(\kappa)}^{2}
\end{aligned}
$$

by using (3.3) and (3.4), we obtain:

$$
\begin{aligned}
\left\|\chi_{h}^{n}\right\|_{0, \kappa}^{2}+\frac{k \mu_{p}}{2} \sum_{i=1}^{n}\left\|\chi_{h}^{i}\right\|_{0, \kappa}^{2} & \lesssim k n h_{\kappa}^{2 \sigma}\left(\left\|\omega^{n}\right\|_{H^{\sigma}(\kappa)}+\left\|\partial_{t} \omega^{n}\right\|_{H^{\sigma}(\kappa)}\right)^{2} \\
& +\frac{3 k(\beta g)^{2}}{2 \mu_{p}} \sum_{i=1}^{n}\left\|T^{i}-T_{h}^{i}\right\|_{H^{1}(\kappa)}^{2} .
\end{aligned}
$$

Finally, using the Jensen's inequality, we get:

$$
\begin{aligned}
\left\|\chi_{h}^{n}\right\|_{L^{2}(\Omega)}^{2}+\frac{k \mu_{p}}{2} \sum_{i=1}^{n}\left\|\chi_{h}^{i}\right\|_{L^{2}(\Omega)}^{2} \lesssim & k n h^{2 \sigma}\left(\|\omega\|_{L^{\infty}\left(0, T, H^{\sigma}(\Omega)\right)}^{2}+\|\omega\|_{W^{1, \infty}\left(0, T, H^{\sigma}(\Omega)\right)}^{2}\right) \\
+ & \frac{3 k(\beta g)^{2}}{2 \mu_{p}} \sum_{i=1}^{n}\left\|T^{i}-T_{h}^{i}\right\|_{H^{1}(\Omega)}^{2} .
\end{aligned}
$$

We conclude, using the triangular inequality

$$
\left\|\omega^{n}-\omega_{h}^{n}\right\|_{L^{2}(\Omega)}^{2}+\frac{k \mu_{p}}{2} \sum_{i=1}^{n}\left\|\omega^{i}-\omega_{h}^{i}\right\|_{L^{2}(\Omega)} \lesssim k n h^{2 \sigma}+\frac{3 k(\beta g)^{2}}{2 \mu_{p}} \sum_{i=1}^{n}\left\|T^{i}-T_{h}^{i}\right\|_{H^{1}(\Omega)}^{2} .
$$

we have the following error estimate for the stream function

Lemma 3.2. For any $\psi^{n}$ and $\psi_{h}^{n}$ solution of the problem $\left(\mathcal{P}_{v}^{n}\right)$ and $\left(\mathcal{P}_{h}^{n}\right)$ respectively, we have:

$$
\left\|\psi^{n}-\psi_{h}^{n}\right\|_{W^{1,4}(\Omega)} \lesssim h^{\sigma}\|\psi\|_{L^{\infty}\left(0, t, W^{1+\sigma, 4}(\Omega)\right)}+\left\|\omega^{n}-\omega_{h}^{n}\right\|_{L^{2}(\Omega)} .
$$


Proof. Let $\psi^{n}$ and $\psi_{h}^{n}$ be the solution of the problem $\left(\mathcal{P}_{v}^{n}\right)$ and $\left(\mathcal{P}_{h}^{n}\right)$ respectively. From $[14,15]$ we have:

$$
\left|\psi^{n}-\psi_{h}^{n}\right|_{W^{1,4}(\Omega)} \lesssim \sup _{\substack{\stackrel{\circ}{W}^{1,4}\\}} \frac{\left\langle\nabla\left(\psi^{n}-\psi_{h}^{n}\right), \nabla v\right\rangle}{|v|_{W^{1, \frac{4}{3}}(\Omega)}}
$$

However

$$
\left\langle\nabla\left(\psi^{n}-\psi_{h}^{n}\right), \nabla v\right\rangle=\left\langle\nabla\left(\psi^{n}-\psi_{h}^{n}\right), \nabla\left(v-\Pi_{h} v\right)\right\rangle+\left\langle\nabla\left(\psi^{n}-\psi_{h}^{n}\right), \nabla\left(\Pi_{h} v\right)\right\rangle,
$$

where $\Pi_{h}$ is the projection operator verifying(2.6). Moreover, we have

$$
\left\langle\nabla\left(R_{h} \psi^{n}-\psi_{h}^{n}\right), \nabla\left(\Pi_{h} v-v\right)\right\rangle=0 \text { and }\left\langle\nabla\left(R_{h} \psi^{n}-\psi^{n}\right), \nabla\left(\Pi_{h} v\right)\right\rangle=0 .
$$

Then we get,

$$
\left\langle\nabla\left(\psi^{n}-\psi_{h}^{n}\right), \nabla v\right\rangle=\left\langle\nabla\left(\psi^{n}-R_{h} \psi^{n}\right), \nabla\left(v-\Pi_{h} v\right)\right\rangle+\left\langle\nabla\left(\psi^{n}-\psi_{h}^{n}\right), \nabla\left(\Pi_{h} v\right)\right\rangle .
$$

By choosing $v_{h}=\Pi_{h} v$ as test function in the last equation of the problem $\left(\mathcal{P}_{h}^{n}\right)$, we obtain:

$$
\left\langle\nabla\left(\psi^{n}-\psi_{h}^{n}\right), \nabla v\right\rangle=\left\langle\nabla\left(\psi^{n}-R_{h} \psi^{n}\right), \nabla v\right\rangle+\left\langle\omega^{n}-\omega_{h}^{n}, \Pi_{h} v\right\rangle .
$$

Then we get

$$
\left\langle\nabla\left(\psi^{n}-R_{h} \psi^{n}\right), \nabla v\right\rangle \leq\left|\psi^{n}-R_{h} \psi^{n}\right|_{W^{1,4}(\Omega)}|v|_{W^{1, \frac{4}{3}(\Omega)}},
$$

and

$$
\left\langle\omega^{n}-\omega_{h}^{n}, \Pi_{h} v\right\rangle \leq\left\|\omega^{n}-\omega_{h}^{n}\right\|_{L^{2}(\Omega)}\left\|\Pi_{h} v\right\|_{L^{2}(\Omega)} .
$$

On the other hand, due to the embedding of $W^{1, \frac{4}{3}}(\Omega)$ into $L^{2}(\Omega)$, we have:

$$
\left\|\Pi_{h} v\right\|_{L^{2}(\Omega)} \lesssim\left|\Pi_{h} v\right|_{W^{1, \frac{4}{3}}(\Omega)} .
$$

In addition,

we get:

$$
\left|\Pi_{h} v\right|_{W^{1, \frac{4}{3}}(\Omega)} \lesssim|v|_{W^{1, \frac{4}{3}}(\Omega)},
$$

$$
\sup _{v \in \stackrel{W}{W}^{1,4}(\Omega)} \frac{\left\langle\omega^{n}-\omega_{h}^{n}, \Pi_{h} v\right\rangle}{|v|_{W^{1, \frac{4}{3}}(\Omega)}} \lesssim\left\|\omega^{n}-\omega_{h}^{n}\right\|_{L^{2}(\Omega)}
$$

From the inequalities (3.6), (3.7) and (3.8), we conclude that

$$
\left|\psi^{n}-\psi_{h}^{n}\right|_{W^{1,4}(\Omega)} \lesssim\left|\psi^{n}-R_{h} \psi^{n}\right|_{W^{1,4}(\Omega)}+\left\|\omega^{n}-\omega_{h}^{n}\right\|_{L^{2}(\Omega)} .
$$

However, we can write:

$$
\left\|\psi^{n}-\psi_{h}^{n}\right\|_{W^{1,4}(\Omega)} \lesssim h^{\sigma}\|\psi\|_{L^{\infty}\left(0, t, W^{1+\sigma, 4}(\Omega)\right)}+\left\|\omega^{n}-\omega_{h}^{n}\right\|_{L^{2}(\Omega)} .
$$

For the temperature estimate, we will need the following lemmas:

Lemma 3.3. For all constants $\theta_{3}, \theta_{4}$ independents of $k$ and $h$, we have

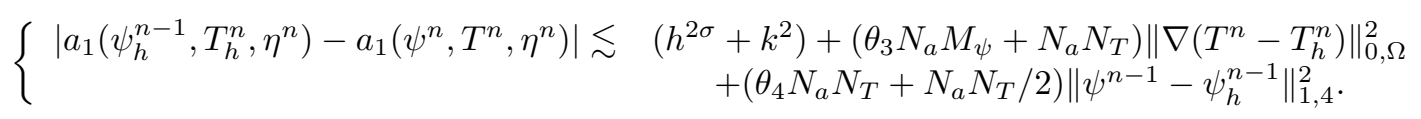

Proof. We set $\eta^{n}=r_{h} T^{n}-T_{h}^{n}$. By using the triangular inequality, we have

$$
\begin{aligned}
\left|a_{1}\left(\psi_{h}^{n-1}, T_{h}^{n}, \eta^{n}\right)-a_{1}\left(\psi^{n}, T^{n}, \eta^{n}\right)\right| \leq & \left|a_{1}\left(\psi_{h}^{n-1}, T_{h}^{n}, \eta^{n}\right)-a_{1}\left(\psi_{h}^{n-1}, T^{n}, \eta^{n}\right)\right| \\
& +\left|a_{1}\left(\psi_{h}^{n-1}, T^{n}, \eta^{n}\right)-a_{1}\left(\psi^{n-1}, T^{n}, \eta^{n}\right)\right| \\
& +\left|a_{1}\left(\psi^{n-1}, T^{n}, \eta^{n}\right)-a_{1}\left(\psi^{n}, T^{n}, \eta^{n}\right)\right| \\
\leq & N_{a} M_{\psi}\left\|\nabla\left(T^{n}-T_{h}^{n}\right)\right\|_{0, \Omega}\left\|\nabla\left(T_{h}^{n}-r_{h} T^{n}\right)\right\|_{0, \Omega} \\
& +N_{a} N_{T}\left\|\psi^{n-1}-\psi_{h}^{n-1}\right\|_{1,4}\left\|\nabla\left(T^{n}-r_{h} T^{n}\right)\right\|_{0, \Omega} \\
& +N_{a} N_{T}\left\|\psi^{n-1}-\psi_{h}^{n-1}\right\|_{1,4}\left\|\nabla\left(T^{n}-T_{h}^{n}\right)\right\|_{0, \Omega} \\
& +N_{a} N_{T}\left\|\psi^{n}-\psi^{n-1}\right\|_{1, \Omega}\left\|\nabla\left(T^{n}-r_{h} T^{n}\right)\right\|_{0, \Omega} \\
& +N_{a} N_{T}\left\|\psi^{n}-\psi^{n-1}\right\|_{1,4}\left\|\nabla\left(T^{n}-T_{h}^{n}\right)\right\|_{0, \Omega} .
\end{aligned}
$$


It leads to

$$
\begin{aligned}
\left|a_{1}\left(\psi_{h}^{n-1}, T_{h}^{n}, \eta^{n}\right)-a_{1}\left(\psi^{n}, T^{n}, \eta^{n}\right)\right| \leq & \left(\theta_{3} N_{a} M_{\psi}+N_{a} N_{T}\right)\left\|\nabla\left(T^{n}-T_{h}^{n}\right)\right\|_{0, \Omega}^{2}+\left\|\nabla\left(T^{n}-r_{h} T^{n}\right)\right\|_{0, \Omega}^{2} \\
& +\left(\theta_{4} N_{a} N_{T}+N_{a} N_{T} / 2\right)\left\|\psi_{h}^{n-1}-\psi^{n-1}\right\|_{1, \Omega}^{2}+\left\|\psi^{n}-\psi^{n-1}\right\|_{1, \Omega}^{2} \\
\lesssim & \left(h^{2 \sigma}+k^{2}\right)+\left(\theta_{3} N_{a} M_{\psi}+N_{a} N_{T}\right)\left\|\nabla\left(T^{n}-T_{h}^{n}\right)\right\|_{0, \Omega}^{2} \\
& +\left(\theta_{4} N_{a} N_{T}+N_{a} N_{T} / 2\right)\left\|\psi^{n-1}-\psi_{h}^{n-1}\right\|_{1,4}^{2} .
\end{aligned}
$$

Lemma 3.4. We have the following a priori estimate:

$$
\left\|\bar{\partial}_{t} r_{h} T^{n}-\partial_{t} T^{n}\right\|_{0} \lesssim\left(h^{\sigma}+k\right)
$$

Proof. By applying the development of Taylor with remainder integral, we obtain

$$
\begin{aligned}
\left\|\bar{\partial}_{t} r_{h} T^{n}-\partial_{t} T^{n}\right\|_{0, \Omega} \leq & \left\|\bar{\partial}_{t} r_{h} T^{n}-\bar{\partial}_{t} T^{n}\right\|_{0, \Omega}+\left\|\bar{\partial}_{t} T^{n}-\partial_{t} T^{n}\right\|_{0, \Omega} \\
= & \left\|\frac{1}{k} \int_{t_{n-1}}^{t_{n}}\left(r_{h} \partial_{t} T(s)-\partial_{t} T(s)\right) d s\right\|_{0, \Omega} \\
& +\left\|\int_{t_{n-1}}^{t_{n}}\left(s-t^{n-1}\right) \partial_{t^{2}}^{2} T(s) d s\right\|_{0, \Omega} \\
\lesssim & \left(h^{\sigma}\|T\|_{W^{1, \infty}\left(0, t, H^{\sigma}(\Omega)\right)}+k\|T\|_{W^{2, \infty}\left(0, t, L^{2}(\Omega)\right)}\right) \\
\lesssim & \left(h^{\sigma}+k\right) .
\end{aligned}
$$

Lemma 3.5. For all constants $\theta_{i}, i=3, . ., 7$ independents of $h$ and $k$, we have

$$
\left\{\begin{aligned}
\frac{1}{2 k}\left(\left\|\eta^{n}\right\|_{0, \Omega}^{2}-\left\|\eta^{n-1}\right\|_{0, \Omega}^{2}+\right. & \left.\left\|\eta^{n}-\eta^{n-1}\right\|_{0, \Omega}^{2}\right)+\lambda\left\|\nabla\left(T^{n}-T_{h}^{n}\right)\right\|_{0, \Omega}^{2} \\
& \lesssim\left(h^{2 \sigma}+k^{2}\right)+\left(\theta_{5}+\theta_{6}+\theta_{7}\right)\left\|\eta^{n}\right\|_{0, \Omega}^{2} \\
& +\left(\theta_{3} N_{a} M_{\psi}+N_{a} N_{T}\right)\left\|\nabla\left(T^{n}-T_{h}^{n}\right)\right\|_{0, \Omega}^{2} \\
& +2 \rho^{2} \frac{N_{C}^{2}\left(\frac{E \alpha}{R T_{i}^{2}}\right)^{2}}{4 \theta_{7}}\left\|\nabla\left(T^{n-1}-T_{h}^{n-1}\right)\right\|_{0, \Omega}^{2} \\
& +\frac{(\alpha \rho)^{2}}{4 \theta_{6}}\left\|\nabla\left(C^{n}-C_{h}^{n}\right)\right\|_{0, \Omega}^{2} \\
& +\left(\theta_{4} N_{a} N_{T}+\frac{N_{a} N_{T}}{2}\right)\left\|\psi^{n-1}-\psi_{h}^{n-1}\right\|_{1,4}^{2} .
\end{aligned}\right.
$$

Proof. For $T^{n}$ and $T_{h}^{n}$ solution of the problem $\left(\mathcal{P}_{h}^{n}\right)$ and $\left(\mathcal{P}_{v}^{n}\right)$, from the tree following equalities:

$$
\begin{gathered}
\left(\bar{\partial}_{t} \eta^{n}, \eta^{n}\right)+\lambda j\left(T^{n}-T_{h}^{n}, T^{n}-T_{h}^{n}\right)=\left(\bar{\partial}_{t} r_{h} T^{n}-\bar{\partial}_{t} T_{h}^{n}, \eta^{n}\right)+\lambda j\left(T^{n}-T_{h}^{n}, T^{n}-T_{h}^{n}\right), \\
\left(\bar{\partial}_{t} T_{h}^{n}, \eta^{n}\right)=-\lambda j\left(T_{h}^{n}, \eta^{n}\right)-a_{1}\left(\psi_{h}^{n-1}, T_{h}^{n}, \eta^{n}\right)+\mathrm{z}\left(C_{h}^{n}, T_{h}^{n-1}, \eta^{n}\right)
\end{gathered}
$$

and

$$
\left(\partial_{t} T^{n}, \eta^{n}\right)=-\lambda j\left(T^{n}, \eta^{n}\right)-a_{1}\left(\psi^{n}, T^{n}, \eta^{n}\right)+\mathrm{z}\left(C^{n}, T^{n}, \eta^{n}\right),
$$

where $\eta^{n}=r_{h} T^{n}-T_{h}^{n}$, we obtain

$$
\begin{aligned}
\left(\bar{\partial}_{t} \eta^{n}, \eta^{n}\right)+\lambda j\left(T^{n}-T_{h}^{n}, T^{n}-T_{h}^{n}\right)= & \left(\bar{\partial}_{t} r_{h} T^{n}-\partial_{t} T^{n}, \eta^{n}\right)+\lambda j\left(T_{h}^{n}-T^{n}, \eta^{n}\right) \\
& +\lambda j\left(T^{n}-T_{h}^{n}, T^{n}-T_{h}^{n}\right)+a_{1}\left(\psi_{h}^{n-1}, T_{h}^{n}, \eta^{n}\right) \\
& -a_{1}\left(\psi^{n}, T^{n}, \eta^{n}\right)+\mathrm{Z}\left(C^{n}, T^{n}, \eta^{n}\right) \\
& -\mathrm{Z}\left(C_{h}^{n}, T_{h}^{n-1}, \eta^{n}\right) .
\end{aligned}
$$

However

$$
\begin{aligned}
j\left(T^{n}-T_{h}^{n}, T^{n}-T_{h}^{n}\right)+j\left(T_{h}^{n}-T^{n}, \eta^{n}\right)= & j\left(T^{n}-T_{h}^{n}, T^{n}-r_{h} T^{n}\right) \\
= & j\left(T^{n}-r_{h} T^{n}, T^{n}-r_{h} T^{n}\right) \\
& +j\left(r_{h} T^{n}-T_{h}^{n}, T^{n}-r_{h} T^{n}\right),
\end{aligned}
$$

using (3.1), the equalities (3.9) and (3.10), yields to

$$
\begin{aligned}
\frac{1}{2 k}\left(\left\|\eta^{n}\right\|_{0, \Omega}^{2}-\left\|\eta^{n-1}\right\|_{0, \Omega}^{2}+\left\|\eta^{n}-\eta^{n-1}\right\|_{0, \Omega}^{2}\right) & +\lambda\left\|\nabla\left(T^{n}-T_{h}^{n}\right)\right\|_{0, \Omega}^{2} \\
& =\left(\bar{\partial}_{t} r_{h} T^{n}-\partial_{t} T^{n}, \eta^{n}\right)+\lambda\left\|\nabla\left(T^{n}-r_{h} T^{n}\right)\right\|_{0, \Omega}^{2} \\
& +a_{1}\left(\psi_{h}^{n-1}, T_{h}^{n}, \eta^{n}\right)-a_{1}\left(\psi^{n}, T^{n}, \eta^{n}\right) \\
& +\mathrm{Z}\left(C^{n}, T^{n}, \eta^{n}\right)-\mathrm{z}\left(C_{h}^{n}, T_{h}^{n-1}, \eta^{n}\right) .
\end{aligned}
$$


We have also

$$
\begin{aligned}
\mathrm{z}\left(C^{n}, T^{n}, \eta^{n}\right)-\mathrm{z}\left(C_{h}^{n}, T_{h}^{n-1}, \eta^{n}\right)= & \mathrm{z}\left(C^{n}, T^{n}, \eta^{n}\right)-\mathrm{z}\left(C^{n}, T^{n-1}, \eta^{n}\right) \\
& +\mathrm{z}\left(C^{n}, T^{n-1}, \eta^{n}\right)-\mathrm{z}\left(C_{h}^{n}, T^{n-1}, \eta^{n}\right) \\
& +\mathrm{z}\left(C_{h}^{n}, T^{n-1}, \eta^{n}\right)-\mathrm{z}\left(C_{h}^{n}, T_{h}^{n-1}, \eta^{n}\right) \\
\lesssim & k\|C\|_{W^{1, \infty}\left(0, t, H^{1}(\Omega)\right.}\left\|\eta^{n}\right\|_{0, \Omega} \\
& +\rho \alpha\left\|\nabla\left(C^{n}-C_{h}^{n}\right)\right\|_{0, \Omega}\left\|\eta^{n}\right\|_{0, \Omega} \\
& +\sqrt{2} \rho \frac{E \alpha}{R T_{i}^{2}} N_{C}\left\|\nabla\left(T^{n-1}-T_{h}^{n-1}\right)\right\|_{0, \Omega}\left\|\eta^{n}\right\|_{0, \Omega},
\end{aligned}
$$

by using the Lemma 3.3, Lemma 3.4 and the equality (3.11), we have

$$
\begin{aligned}
\frac{1}{2 k}\left(\left\|\eta^{n}\right\|_{0, \Omega}^{2}-\left\|\eta^{n-1}\right\|_{0, \Omega}^{2}+\left\|\eta^{n}-\eta^{n-1}\right\|_{0, \Omega}^{2}\right) & +\lambda\left\|\nabla\left(T^{n}-T_{h}^{n}\right)\right\|_{0, \Omega}^{2} \\
& \lesssim\left(h^{\sigma}+k\right)\left\|\eta^{n}\right\|_{0, \Omega}+\lambda\left\|\nabla\left(T^{n}-r_{h} T^{n}\right)\right\|_{0, \Omega}^{2} \\
& +\left(h^{2 \sigma}+k^{2}\right)+\left(\theta_{3} N_{a} M_{\psi}+N_{a} N_{T}\right)\left\|\nabla\left(T^{n}-T_{h}^{n}\right)\right\|_{0, \Omega}^{2} \\
& +\left(\theta_{4} N_{a} N_{T}+\frac{N_{a} N_{T}}{2}\right)\left\|\psi^{n-1}-\psi_{h}^{n-1}\right\|_{1,4}^{2} \\
& +k\left\|\eta^{n}\right\|_{0, \Omega}+\rho \alpha\left\|\nabla\left(C^{n}-C_{h}^{n}\right)\right\|_{0, \Omega}\left\|\eta^{n}\right\|_{0, \Omega} \\
& +\sqrt{2} \rho N_{C} \frac{E \alpha}{R T_{i}^{2}}\left\|\nabla\left(T^{n-1}-T_{h}^{n-1}\right)\right\|_{0, \Omega}\left\|\eta^{n}\right\|_{0, \Omega} .
\end{aligned}
$$

Finally, we deduce

$$
\begin{aligned}
\frac{1}{2 k}\left(\left\|\eta^{n}\right\|_{0, \Omega}^{2}-\left\|\eta^{n-1}\right\|_{0, \Omega}^{2}+\left\|\eta^{n}-\eta^{n-1}\right\|_{0, \Omega}^{2}\right) & +\lambda\left\|\nabla\left(T^{n}-T_{h}^{n}\right)\right\|_{0, \Omega}^{2} \\
& \lesssim\left(h^{2 \sigma}+k^{2}\right)+\left(\theta_{5}+\theta_{6}+\theta_{7}\right)\left\|\eta^{n}\right\|_{0, \Omega}^{2} \\
& +\left(\theta_{3} N_{a} M_{\psi}+N_{a} N_{T}\right)\left\|\nabla\left(T^{n}-T_{h}^{n}\right)\right\|_{0, \Omega}^{2} \\
& +2 \rho^{2} \frac{N_{C}^{2}\left(\frac{E \alpha}{R T_{i}^{2}}\right)^{2}}{4 \theta_{7}}\left\|\nabla\left(T^{n-1}-T_{h}^{n-1}\right)\right\|_{0, \Omega}^{2} \\
& +\left(\theta_{4} N_{a} N_{T}+\frac{N_{a} N_{T}}{2}\right)\left\|\psi^{n-1}-\psi_{h}^{n-1}\right\|_{1,4}^{2} \\
& +\frac{(\alpha \rho)^{2}}{4 \theta_{6}}\left\|\nabla\left(C^{n}-C_{h}^{n}\right)\right\|_{0, \Omega}^{2} .
\end{aligned}
$$

Now, we are able to state the following:

Lemma 3.6. We assume that $\left(k \leq \frac{1}{4}\right), \mathcal{H}_{1}$ and $\mathcal{H}_{4}$ are verified. Then we have

$$
\left\{\begin{aligned}
\left\|T^{n}-T_{h}^{n}\right\|_{0, \Omega}^{2}+\frac{8}{7} \lambda k\left\|\nabla\left(T^{n}-T_{h}^{n}\right)\right\|_{0, \Omega}^{2} \lesssim & \frac{16 k N_{a} N_{T}}{7}\left(\theta_{4}+\frac{1}{2}\right) \sum_{i=1}^{n-1}\left\|\psi^{i}-\psi_{h}^{i}\right\|_{1,4}^{2} \\
& +\frac{48}{7}(\alpha \rho)^{2} k \sum_{i=1}^{n}\left\|\nabla\left(C^{i}-C_{h}^{i}\right)\right\|_{0, \Omega}^{2} \\
& k n\left(h^{2 \sigma}+k^{2}\right) .
\end{aligned}\right.
$$

For the proof of this lemma, we recall the following lemma, known as the Gronwal lemma [16]:

Lemma 3.7. Let $a_{n}, b_{n}$ and $c_{n}$ three positive sequences, $c_{n}$ not decreasing sequence, Assume that

$$
a_{n}+b_{n} \leq c_{n}+\bar{\nu} \sum_{i=0}^{n-1} a_{i}, \bar{\nu}>0
$$

and

$$
a_{0}+b_{0} \leq c_{0}
$$

We have the following result:

$$
a_{n} \leq c_{n} \exp (\bar{\nu} n)
$$


Proof. By choosing $\theta_{5}=\theta_{6}=\theta_{7}=\frac{1}{12}$ and $\theta_{3}=\frac{\lambda-2 N_{a} N_{T}}{2 N_{a} M_{\psi}}$ in Lemma 3.5, we obtain

$$
\begin{aligned}
\frac{1}{2 k}\left(\left\|\eta^{n}\right\|_{0, \Omega}^{2}-\left\|\eta^{n-1}\right\|_{0, \Omega}^{2}+\left\|\eta^{n}-\eta^{n-1}\right\|_{0, \Omega}^{2}\right) & +\frac{\lambda}{2}\left\|\nabla\left(T^{n}-T_{h}^{n}\right)\right\|_{0, \Omega}^{2} \\
& \lesssim \frac{1}{4}\left\|\eta^{n}\right\|_{0, \Omega}^{2}+\left(h^{2 \sigma}+k^{2}\right)++3(\alpha \rho)^{2}\left\|\nabla\left(C^{n}-C_{h}^{n}\right)\right\|_{0, \Omega}^{2} \\
& +6 \rho^{2} N_{C}^{2}\left(\frac{E \alpha}{R T_{i}^{2}}\right)^{2}\left\|\nabla\left(T^{n-1}-T_{h}^{n-1}\right)\right\|_{0, \Omega}^{2} \\
& +\left(\theta_{4} N_{a} N_{T}+\frac{N_{a} N_{T}}{2}\right)\left\|\psi^{n-1}-\psi_{h}^{n-1}\right\|_{1,4}^{2} .
\end{aligned}
$$

Then, by summing over time, we have

$$
\begin{aligned}
\frac{1}{2 k}\left\|\eta^{n}\right\|_{0, \Omega}^{2}+\frac{\lambda}{2} \sum_{i=1}^{n}\left\|\nabla\left(T^{i}-T_{h}^{i}\right)\right\|_{0, \Omega}^{2} \lesssim & \frac{1}{4} \sum_{i=1}^{n}\left\|\eta^{i}\right\|_{0, \Omega}^{2}+n\left(h^{2 \sigma}+k^{2}\right) \\
& +6 \rho^{2} N_{C}^{2}\left(\frac{E \alpha}{R T_{i}^{2}}\right)^{2} \sum_{i=1}^{n-1}\left\|\nabla\left(T^{i}-T_{h}^{i}\right)\right\|_{0, \Omega}^{2} \\
& +3(\alpha \rho)^{2} \sum_{i=1}^{n}\left\|\nabla\left(C^{i}-C_{h}^{i}\right)\right\|_{0, \Omega}^{2} \\
& +\left(\theta_{4} N_{a} N_{T}+\frac{N_{a} N_{T}}{2}\right) \sum_{i=1}^{n-1}\left\|\psi^{i}-\psi_{h}^{i}\right\|_{1,4}^{2} .
\end{aligned}
$$

However, assuming that

$$
6 \rho^{2} N_{C}^{2}\left(\frac{E \alpha}{R T_{i}^{2}}\right)^{2} \leq \frac{\lambda}{2} \text { and } k \leq \frac{1}{4}
$$

then, the following results holds

$$
\begin{aligned}
\frac{7}{8}\left\|\eta^{n}\right\|_{0, \Omega}^{2}+\lambda k\left\|\nabla\left(T^{n}-T_{h}^{n}\right)\right\|_{0, \Omega}^{2} \lesssim & \frac{k}{2} \sum_{i=1}^{n-1}\left\|\eta^{i}\right\|_{0, \Omega}^{2}+k n\left(h^{2 \sigma}+k^{2}\right) \\
& +6(\alpha \rho)^{2} k \sum_{i=1}^{n}\left\|\nabla\left(C^{i}-C_{h}^{i}\right)\right\|_{0, \Omega}^{2} \\
& +2 k\left(\theta_{4} N_{a} N_{T}+\frac{N_{a} N_{T}}{2}\right) \sum_{i=1}^{n-1}\left\|\psi^{i}-\psi_{h}^{i}\right\|_{1,4}^{2}
\end{aligned}
$$

Using the discrete Gronwall Lemma, we have

$$
\begin{aligned}
\left\|\eta^{n}\right\|_{0, \Omega}^{2}+\frac{8}{7} k \lambda\left\|\nabla\left(T^{n}-T_{h}^{n}\right)\right\|_{0, \Omega}^{2} \lesssim & \left(k n\left(h^{2 \sigma}+k^{2}\right)+\frac{16 k}{7}\left(\theta_{4} N_{a} N_{T}+\frac{N_{a} N_{T}}{2}\right) \sum_{i=1}^{n-1}\left\|\psi^{i}-\psi_{h}^{i}\right\|_{1,4}^{2}\right. \\
& \left.+\frac{48}{7}(\alpha \rho)^{2} k \sum_{i=1}^{n}\left\|\nabla\left(C^{i}-C_{h}^{i}\right)\right\|_{0, \Omega}^{2}\right) \exp (k n) .
\end{aligned}
$$

From the triangular inequality

$$
\begin{aligned}
\left\|T^{n}-T_{h}^{n}\right\|_{0, \Omega}^{2}+\frac{8}{7} k \lambda\left\|\nabla\left(T^{n}-T_{h}^{n}\right)\right\|_{0, \Omega}^{2} & \leq\left\|T^{n}-r_{h} T^{n}\right\|_{0, \Omega}^{2}+\left\|\eta^{n}\right\|_{0, \Omega}^{2}+\frac{8}{7} k \lambda\left\|\nabla\left(T^{n}-T_{h}^{n}\right)\right\|_{0, \Omega}^{2} \\
& \lesssim h^{2 \sigma}\|T\|_{L^{\infty}\left(0, t, H^{\sigma}(\Omega)\right)}^{2}+\left\|\eta^{n}\right\|_{0, \Omega}^{2}+\frac{8}{7} k \lambda\left\|\nabla\left(T^{n}-T_{h}^{n}\right)\right\|_{0, \Omega}^{2}
\end{aligned}
$$

The estimate error on concentration derive from the two technical lemmas:

Lemma 3.8. For all constants $\theta_{8}, \theta_{9}$, independents of $h$ and $k$, we have

$$
\left\{\begin{aligned}
\left|a_{1}\left(\psi_{h}^{n-1}, C_{h}^{n}, \epsilon_{1}^{n}\right)-a_{1}\left(\psi^{n}, C^{n}, \epsilon_{1}^{n}\right)\right| \lesssim \quad & \left(\theta_{8} N_{a} M_{\psi}+N_{a} N_{C}\right)\left\|\nabla\left(C^{n}-C_{h}^{n}\right)\right\|_{0, \Omega}^{2} \\
& +N_{a} N_{C}\left(\theta_{9}+\frac{1}{2}\right)\left\|\psi^{n-1}-\psi_{h}^{n-1}\right\|_{1,4}^{2} \\
& \left(h^{2 \sigma}+k^{2}\right) .
\end{aligned}\right.
$$

Proof. The proof is similar to the proof of the Lemma 3.3. 
Lemma 3.9. We have the following estimate:

$$
\left\|\partial_{t} r_{h} C^{n}-\partial_{t} C^{n}\right\|_{0, \Omega} \lesssim\left(h^{\sigma}+k\right)
$$

Proof. The proof is similar to the proof of the Lemma 3.4.

Lemma 3.10. For all constants $\theta_{i}, i=8, . ., 12$ independents of $h$ and $k$, we have

$$
\left\{\begin{array}{cl}
\frac{1}{2 k}\left(\left\|\epsilon^{n}\right\|_{0, \Omega}^{2}-\left\|\epsilon^{n-1}\right\|_{0, \Omega}^{2}+\right. & \left.\left\|\epsilon^{n}-\epsilon^{n-1}\right\|_{0, \Omega}^{2}\right)+\eta\left\|\nabla\left(C^{n}-C_{h}^{n}\right)\right\|_{0, \Omega}^{2} \\
& \lesssim\left(h^{2 \sigma}+k^{2}\right)+\left(\theta_{10}+\theta_{11}+\theta_{12}\right)\left\|\epsilon^{n}\right\|_{0, \Omega}^{2} \\
& +N_{a} N_{C}\left(\theta_{9}+\frac{1}{2}\right)\left\|\psi^{n-1}-\psi_{h}^{n-1}\right\|_{1,4}^{2} \\
& +N_{a}\left(\theta_{8} M_{\psi}+N_{C}\right)\left\|\nabla\left(C^{n}-C_{h}^{n}\right)\right\|_{0, \Omega}^{2} \\
& +2 \rho^{2} \frac{N_{C}^{2}\left(\frac{E \alpha}{R T_{i}^{2}}\right)^{2}}{4 \theta_{12}}\left\|\nabla\left(T^{n}-T_{h}^{n-1}\right)\right\|_{0, \Omega}^{2} \\
& +\frac{(\alpha \rho)^{2}}{4 \theta_{11}}\left\|\nabla\left(C^{n}-C_{h}^{n}\right)\right\|_{0, \Omega}^{2} .
\end{array}\right.
$$

Proof. We set $\epsilon^{n}=r_{h} C^{n}-C_{h}^{n}$. First of all, we have

$$
\left(\bar{\partial}_{t} \epsilon^{n}, \epsilon^{n}\right)+\eta j\left(C^{n}-C_{h}^{n}, C^{n}-C_{h}^{n}\right)=\left(\bar{\partial}_{t} r_{h} C^{n}-\bar{\partial}_{t} C_{h}^{n}, \epsilon^{n}\right)+\eta j\left(C^{n}-C_{h}^{n}, C^{n}-C_{h}^{n}\right),
$$

however

$$
\left(\bar{\partial}_{t} C_{h}^{n}, \epsilon^{n}\right)=-\eta j\left(C_{h}^{n}, \epsilon^{n}\right)-a_{1}\left(\psi_{h}^{n-1}, C_{h}^{n}, \epsilon^{n}\right)-\mathrm{z}\left(C_{h}^{n}, T_{h}^{n-1}, \epsilon^{n}\right) .
$$

Therefore, we get:

$$
\begin{aligned}
\left(\bar{\partial}_{t} \epsilon^{n}, \epsilon^{n}\right)+\eta j\left(C^{n}-C_{h}^{n}, C^{n}-C_{h}^{n}\right)= & \left(\bar{\partial}_{t} r_{h} C^{n}, \epsilon^{n}\right)+\eta j\left(C_{h}^{n}, \epsilon^{n}\right) \\
& +\eta j\left(C^{n}-C_{h}^{n}, C^{n}-C_{h}^{n}\right) \\
& +a_{1}\left(\psi_{h}^{n-1}, C_{h}^{n}, \epsilon^{n}\right)+\mathrm{z}\left(C_{h}^{n}, T_{h}^{n-1}, \epsilon^{n}\right) \\
= & \left(\bar{\partial}_{t} r_{h} C^{n}-\partial_{t} C^{n}, \epsilon^{n}\right)+\eta j\left(C_{h}^{n}-C^{n}, \epsilon^{n}\right) \\
& +\eta j\left(C^{n}-C_{h}^{n}, C^{n}-C_{h}^{n}\right) \\
& +a_{1}\left(\psi_{h}^{n-1}, C_{h}^{n}, \epsilon^{n}\right)-a_{1}\left(\psi^{n}, C^{n}, \epsilon^{n}\right) \\
& -\mathrm{z}\left(C^{n}, T^{n}, \epsilon^{n}\right)+\mathrm{Z}\left(C_{h}^{n}, T_{h}^{n-1}, \epsilon^{n}\right) .
\end{aligned}
$$

Also

$$
\begin{aligned}
j\left(C^{n}-C_{h}^{n}, C^{n}-C_{h}^{n}\right)+j\left(C_{h}^{n}-C^{n}, \epsilon^{n}\right)= & j\left(C^{n}-C_{h}^{n}, C^{n}-r_{h} C^{n}\right) \\
= & j\left(C^{n}-r_{h} C^{n}, C^{n}-r_{h} C^{n}\right) \\
& +j\left(r_{h} C^{n}-C_{h}^{n}, C^{n}-r_{h} C^{n}\right),
\end{aligned}
$$

whence, $j\left(r_{h} C^{n}-C_{h}^{n}, C^{n}-r_{h} C^{n}\right)=0$, by using the identity (3.1), it follows

$$
\begin{aligned}
\frac{1}{2 k}\left(\left\|\epsilon^{n}\right\|^{2}-\left\|\epsilon^{n-1}\right\|^{2}+\left\|\epsilon^{n}-\epsilon^{n-1}\right\|^{2}\right)+ & \eta\left\|\nabla\left(C^{n}-C_{h}^{n}\right)\right\|^{2} \\
= & \left(\bar{\partial}_{t} r_{h} C^{n}-\partial_{t} \psi^{n}, \epsilon^{n}\right)+\eta\left\|\nabla\left(C^{n}-r_{h} C^{n}\right)\right\|_{0, \Omega}^{2} \\
& +a_{1}\left(\psi_{h}^{n-1}, C_{h}^{n}, \epsilon^{n}\right)-a_{1}\left(\psi^{n}, C^{n}, \epsilon^{n}\right) \\
& -\mathrm{z}\left(C^{n}, T^{n}, \epsilon^{n}\right)+\mathrm{z}\left(C_{h}^{n}, T_{h}^{n-1}, \epsilon^{n}\right) .
\end{aligned}
$$

We have as well, using the Holder inequality

$$
\begin{aligned}
\mathrm{z}\left(C^{n}, T^{n}, \epsilon^{n}\right)-\mathrm{z}\left(C_{h}^{n}, T_{h}^{n-1}, \epsilon^{n}\right)= & \mathrm{z}\left(C^{n}, T^{n}, \epsilon^{n}\right)-\mathrm{z}\left(C^{n}, T^{n-1}, \epsilon^{n}\right) \\
& +\mathrm{z}\left(C^{n}, T^{n-1}, \epsilon^{n}\right)-\mathrm{z}\left(C_{h}^{n}, T^{n-1}, \epsilon^{n}\right) \\
& +\mathrm{z}\left(C_{h}^{n}, T^{n-1}, \epsilon^{n}\right)-\mathrm{z}\left(C_{h}^{n}, T_{h}^{n-1}, \epsilon^{n}\right) \\
\lesssim & k\|T\|_{W^{1, \infty}\left(0, t, H^{\sigma}(\Omega)\right)}\left\|\epsilon^{n}\right\|_{0, \Omega} \\
& +\rho \alpha\left\|\nabla\left(C^{n}-C_{h}^{n}\right)\right\|_{0, \Omega}\left\|\epsilon^{n}\right\|_{0, \Omega} \\
& +\sqrt{2} \rho \frac{E \alpha}{R T_{i}^{2}} N_{C}\left\|\nabla\left(T^{n-1}-T_{h}^{n-1}\right)\right\|_{0, \Omega}\left\|\epsilon^{n}\right\|_{0, \Omega},
\end{aligned}
$$


it follows

$$
\begin{aligned}
\frac{1}{2 k}\left(\left\|\epsilon^{n}\right\|_{0, \Omega}^{2}-\left\|\epsilon^{n-1}\right\|_{0, \Omega}^{2}\right. & \left.+\left\|\epsilon^{n}-\epsilon^{n-1}\right\|_{0, \Omega}^{2}\right)+\eta\left\|\nabla\left(C^{n}-C_{h}^{n}\right)\right\|_{0, \Omega}^{2} \\
& \lesssim\left(h^{\sigma}+k\right)\left\|\epsilon^{n}\right\|_{0, \Omega}+\eta\left\|\nabla\left(C^{n}-r_{h} C^{n}\right)\right\|_{0, \Omega}^{2} \\
& +\left(\theta_{8} N_{a} M_{\psi}+N_{a} N_{C}\right)\left\|\nabla\left(C^{n}-C_{h}^{n}\right)\right\|_{0, \Omega}^{2} \\
& +\left(\theta_{9} N_{a} N_{C}+\frac{N_{a} N_{C}}{2}\right)\left\|\psi^{n-1}-\psi_{h}^{n-1}\right\|_{1,4}^{2} \\
& +k\left\|\epsilon^{n}\right\|_{0, \Omega}+\rho \alpha\left\|\nabla\left(C^{n}-C_{h}^{n}\right)\right\|_{0, \Omega}\left\|\epsilon^{n}\right\|_{0, \Omega} \\
& +\sqrt{2} \rho N_{C} \frac{E \alpha}{R T_{i}^{2}}\left\|\nabla\left(T^{n-1}-T_{h}^{n-1}\right)\right\|_{0, \Omega}\left\|\epsilon^{n}\right\|_{0, \Omega} \\
& +\left(h^{2 \sigma}+k^{2}\right) .
\end{aligned}
$$

From where

$$
\begin{aligned}
\frac{1}{2 k}\left(\left\|\epsilon^{n}\right\|_{0, \Omega}^{2}-\left\|\epsilon^{n-1}\right\|_{0, \Omega}^{2}\right. & \left.+\left\|\epsilon^{n}-\epsilon^{n-1}\right\|_{0, \Omega}^{2}\right)+\eta\left\|\nabla\left(C^{n}-C_{h}^{n}\right)\right\|_{0, \Omega}^{2} \\
& \lesssim\left(h^{2 \sigma}+k^{2}\right)+\left(\theta_{10}+\theta_{11}+\theta_{12}\right)\left\|\epsilon^{n}\right\|_{0, \Omega}^{2} \\
& +\left(\theta_{8} N_{a} M_{\psi}+N_{a} N_{C}\right)\left\|\nabla\left(C^{n}-C_{h}^{n}\right)\right\|_{0, \Omega}^{2} \\
& +2 \rho^{2} \frac{N_{C}^{2}\left(\frac{E \alpha}{R T_{i}^{2}}\right)^{2}}{4 \theta_{12}}\left\|\nabla\left(T^{n-1}-T_{h}^{n-1}\right)\right\|_{0, \Omega}^{2} \\
& +\left(\theta_{9} N_{a} N_{C}+\frac{N_{a} N_{C}}{2}\right)\left\|\psi^{n-1}-\psi_{h}^{n-1}\right\|_{1,4}^{2} \\
& +\frac{(\alpha \rho)^{2}}{4 \theta_{11}}\left\|\nabla\left(C^{n}-C_{h}^{n}\right)\right\|_{0, \Omega}^{2} .
\end{aligned}
$$

Lemma 3.11. Assuming that $\left(k \leq \frac{1}{4}\right), \mathcal{H}_{2}$ and $\mathcal{H}_{3}$ are verified, the following estimate holds

$$
\left\{\begin{aligned}
\left\|C^{n}-C_{h}^{n}\right\|_{0, \Omega}^{2}+\frac{8}{7} k(\eta- & \left.6(\alpha \rho)^{2}\right)\left\|\nabla\left(C^{n}-C_{h}^{n}\right)\right\|_{0, \Omega}^{2} \\
& \lesssim k n\left(h^{2 \sigma}+k^{2}\right) \\
& +\frac{16 k N_{a} N_{C}}{7}\left(\theta_{9}+\frac{1}{2}\right) \sum_{i=1}^{n-1}\left\|\psi^{i}-\psi_{h}^{i}\right\|_{1,4}^{2} \\
& +\frac{96}{7} \rho^{2} k N_{C}^{2}\left(\frac{E \alpha}{R T_{i}^{2}}\right)^{2} \sum_{i=1}^{n}\left\|\nabla\left(T^{i}-T_{h}^{i}\right)\right\|_{0, \Omega}^{2}
\end{aligned}\right.
$$

Proof. By choosing

$$
\theta_{10}=\theta_{11}=\theta_{12}=\frac{1}{12}
$$

and

$$
\theta_{8}=\frac{\eta-2 N_{a} N_{C}}{2 N_{a} M_{\psi}} \text { because } N_{a} N_{C}<\frac{\eta}{2},
$$

the inequality in Lemma 3.10 becomes

$$
\begin{aligned}
\frac{1}{2 k}\left(\left\|\epsilon^{n}\right\|_{0, \Omega}^{2}-\left\|\epsilon^{n-1}\right\|_{0, \Omega}^{2}+\left\|\epsilon^{n}-\epsilon^{n-1}\right\|_{0, \Omega}^{2}\right)+ & \frac{\eta}{2}\left\|\nabla\left(C^{n}-C_{h}^{n}\right)\right\|_{0, \Omega}^{2} \\
& \lesssim \frac{1}{4}\left\|\epsilon^{n}\right\|_{0, \Omega}^{2}+\left(h^{2 \sigma}+k^{2}\right) \\
& +6 \rho^{2} N_{C}^{2}\left(\frac{E \alpha}{R T_{i}^{2}}\right)^{2}\left\|\nabla\left(T^{n-1}-T_{h}^{n-1}\right)\right\|_{0, \Omega}^{2} \\
& +N_{a} N_{T}\left(\theta_{4}+\frac{1}{2}\right)\left\|\psi^{n-1}-\psi_{h}^{n-1}\right\|_{1,4}^{2} \\
& +3(\alpha \rho)^{2}\left\|\nabla\left(C^{n}-C_{h}^{n}\right)\right\|_{0, \Omega}^{2} .
\end{aligned}
$$

From where

$$
\begin{aligned}
\frac{1}{2 k}\left\|\epsilon^{n}\right\|_{0, \Omega}^{2}+\frac{\eta}{2} \sum_{i=1}^{n}\left\|\nabla\left(C^{i}-C_{h}^{i}\right)\right\|_{0, \Omega}^{2} \lesssim & \frac{1}{4} \sum_{i=1}^{n}\left\|\epsilon^{i}\right\|_{0, \Omega}^{2}+n\left(h^{2 \sigma}+k^{2}\right) \\
& +6 \rho^{2} N_{C}^{2}\left(\frac{E \alpha}{R T_{i}^{2}}\right)^{2} \sum_{i=1}^{n-1}\left\|\nabla\left(T^{i}-T_{h}^{i}\right)\right\|_{0, \Omega}^{2} \\
& +3(\alpha \rho)^{2} \sum_{i=1}^{n}\left\|\nabla\left(C^{i}-C_{h}^{i}\right)\right\|_{0, \Omega}^{2} \\
& +N_{a} N_{C}\left(\theta_{9}+\frac{1}{2}\right) \sum_{i=1}^{n-1}\left\|\psi^{i}-\psi_{h}^{i}\right\|_{1,4}^{2} .
\end{aligned}
$$


While multiplying by $2 k$, using the assumptions $\left(k \leq \frac{1}{4}\right)$ and $\left(6(\alpha \rho)^{2}<\eta\right)$, we obtain

$$
\begin{aligned}
\frac{7}{8}\left\|\epsilon^{n}\right\|_{0, \Omega}^{2}+k\left(\eta-6(\alpha \rho)^{2}\right) & \left\|\nabla\left(C^{n}-C_{h}^{n}\right)\right\|_{0, \Omega}^{2} \\
& \lesssim \frac{k}{2} \sum_{i=1}^{n-1}\left\|\epsilon^{i}\right\|_{0, \Omega}^{2}+k n\left(h^{2 \sigma}+k^{2}\right) \\
& +12 \rho^{2} k N_{C}^{2}\left(\frac{E \alpha}{R T_{i}^{2}}\right)^{2} \sum_{i=1}^{n}\left\|\nabla\left(T^{i}-T_{h}^{i}\right)\right\|_{0, \Omega}^{2} \\
& +2 k N_{a} N_{C}\left(\theta_{9}+\frac{1}{2}\right) \sum_{i=1}^{n-1}\left\|\psi^{i}-\psi_{h}^{i}\right\|_{1,4}^{2} .
\end{aligned}
$$

It leads to

$$
\begin{aligned}
\left\|\epsilon^{n}\right\|_{0, \Omega}^{2}+\frac{8}{7} k\left(\eta-6(\alpha \rho)^{2}\right) & \left\|\nabla\left(C^{n}-C_{h}^{n}\right)\right\|_{0, \Omega}^{2} \\
& \lesssim \frac{4 k}{7} \sum_{i=1}^{n-1}\left\|\epsilon^{i}\right\|_{0, \Omega}^{2}+k n\left(h^{2 \sigma}+k^{2}\right) \\
+ & \frac{96}{7} \rho^{2} k N_{C}^{2}\left(\frac{E \alpha}{R T_{i}^{2}}\right)^{2} \sum_{i=1}^{n}\left\|\nabla\left(T^{i}-T_{h}^{i}\right)\right\|_{0, \Omega}^{2} \\
+ & \frac{16 k N_{a} N_{C}}{7}\left(\theta_{9}+\frac{1}{2}\right) \sum_{i=1}^{n-1}\left\|\psi^{i}-\psi_{h}^{i}\right\|_{1,4}^{2} .
\end{aligned}
$$

Using the discrete lemma of Gronwall

$$
\begin{aligned}
\left\|\epsilon^{n}\right\|_{0, \Omega}^{2}+\frac{8}{7} k\left(\eta-6(\alpha \rho)^{2}\right) & \left\|\nabla\left(C^{n}-C_{h}^{n}\right)\right\|_{0, \Omega}^{2} \\
& \lesssim\left(k n\left(h^{2 \sigma}+k^{2}\right)+\frac{96}{7} \rho^{2} k N_{C}^{2}\left(\frac{E \alpha}{R T_{i}^{2}}\right)^{2} \sum_{i=1}^{n}\left\|\nabla\left(T^{i}-T_{h}^{i}\right)\right\|_{0, \Omega}^{2}\right. \\
& \left.+\frac{16 k N_{a} N_{C}}{7}\left(\theta_{9}+\frac{1}{2}\right) \sum_{i=1}^{n-1}\left\|\psi^{i}-\psi_{h}^{i}\right\|_{1,4}^{2}\right) \exp (k n) .
\end{aligned}
$$

By using the triangular inequality, we get

$$
\begin{aligned}
\left\|C^{n}-C_{h}^{n}\right\|_{0, \Omega}^{2} & +\frac{8}{7} k\left(\eta-6(\alpha \rho)^{2}\right)\left\|\nabla\left(C^{n}-C_{h}^{n}\right)\right\|_{0, \Omega}^{2} \\
& \leq\left\|C^{n}-r_{h} C^{n}\right\|_{0, \Omega}^{2}+\left\|\epsilon^{n}\right\|_{0, \Omega}^{2} \\
& +\frac{8}{7} k\left(\eta-6(\alpha \rho)^{2}\right)\left\|\nabla\left(C^{n}-C_{h}^{n}\right)\right\|_{0, \Omega}^{2} \\
& \lesssim h^{2 \sigma}\|C\|_{L^{\infty}\left(0, t, H^{\sigma}(\Omega)\right)}^{2}+\left\|\epsilon^{n}\right\|_{0, \Omega}^{2} \\
& +\frac{8}{7} k\left(\eta-6(\alpha \rho)^{2}\right)\left\|\nabla\left(C^{n}-C_{h}^{n}\right)\right\|_{0, \Omega}^{2} .
\end{aligned}
$$

We are able now to establish the error estimate on the stream function:

Lemma 3.12. supposing that $\mathcal{H}_{5}$ is verified, then for $\theta_{4}>0$ and $\theta_{9}>0$, we have the following error estimate

$$
\left\|\psi^{n}-\psi_{h}^{n}\right\|_{W(\Omega, 4}^{2} \lesssim e^{k^{2} n^{2} \frac{3(\beta g)^{2}}{2 \mu_{p}} \frac{16 N_{a} k}{7(1-w)}\left(N_{T}\left(\theta_{4}+\frac{1}{2}\right)+N_{C}\left(\theta_{9}+\frac{1}{2}\right)\right)}\left(k n\left(h^{2 \sigma}+k^{2}\right)+k^{2} n^{2}\left(h^{2 \sigma}+k^{2}\right)\right) .
$$

Proof. According to the Lemmas 3.11 and 3.6, we have

$$
\begin{aligned}
\left\|T^{n}-T_{h}^{n}\right\|_{0, \Omega}^{2}+ & \frac{8}{7} k \lambda\left\|\nabla\left(T^{n}-T_{h}^{n}\right)\right\|_{0, \Omega}^{2} \\
& \lesssim k n\left(h^{2 \sigma}+k^{2}\right) \\
& +\frac{16 k N_{a} N_{T}}{7}\left(\theta_{4}+\frac{1}{2}\right) \sum_{i=1}^{n-1}\left\|\psi^{i}-\psi_{h}^{i}\right\|_{1,4}^{2} \\
& +\frac{48 k}{7}(\alpha \rho)^{2} \sum_{i=1}^{n}\left\|\nabla\left(C^{i}-C_{h}^{i}\right)\right\|_{0, \Omega}^{2}
\end{aligned}
$$


and

$$
\begin{aligned}
\left\|C^{n}-C_{h}^{n}\right\|_{0, \Omega}^{2} & +\frac{8}{7} k\left(\eta-6(\alpha \rho)^{2}\right)\left\|\nabla\left(C^{n}-C_{h}^{n}\right)\right\|_{0, \Omega}^{2} \\
& \lesssim k n\left(h^{2 \sigma}+k^{2}\right) \\
& +\frac{16 k N_{a} N_{C}}{7}\left(\theta_{9}+\frac{1}{2}\right) \sum_{i=1}^{n-1}\left\|\psi^{i}-\psi_{h}^{i}\right\|_{1,4}^{2} \\
& +\frac{8}{7}\left(12 \rho^{2} k\right)\left(N_{C} \frac{E \alpha}{R T_{i}^{2}}\right)^{2} \sum_{i=1}^{n}\left\|\nabla\left(T^{i}-T_{h}^{i}\right)\right\|_{0, \Omega}^{2} .
\end{aligned}
$$

By summing the inequality (3.12) and (3.13), we obtain

$$
\begin{gathered}
\left\|T^{n}-T_{h}^{n}\right\|_{0, \Omega}^{2}+\left\|C^{n}-C_{h}^{n}\right\|_{0, \Omega}^{2}+\frac{8}{7} \lambda k\left\|\nabla\left(T^{n}-T_{h}^{n}\right)\right\|_{0, \Omega}^{2}+\frac{8}{7} k\left(\eta-6(\alpha \rho)^{2}\right)\left\|\nabla\left(C^{n}-C_{h}^{n}\right)\right\|_{0, \Omega}^{2} \\
\lesssim k n\left(h^{2 \sigma}+k^{2}\right)+\frac{16 k N_{a}}{7}\left(N_{T}\left(\theta_{4}+\frac{1}{2}\right)+N_{C}\left(\theta_{9}+\frac{1}{2}\right)\right) \sum_{i=1}^{n-1}\left\|\psi^{i}-\psi_{h}^{i}\right\|_{1,4}^{2} \\
+\frac{48}{7} \rho^{2} k\left(\alpha^{2} \sum_{i=1}^{n}\left\|\nabla\left(C^{i}-C_{h}^{i}\right)\right\|_{0, \Omega}^{2}+2\left(N_{C} \frac{E \alpha}{R T_{i}^{2}}\right)^{2} \sum_{i=1}^{n}\left\|\nabla\left(T^{i}-T_{h}^{i}\right)\right\|_{0, \Omega}^{2}\right),
\end{gathered}
$$

if we take

$$
w=\frac{48}{7} \rho^{2} k\left(\max \left(\alpha^{2}, 2 N_{C}^{2}\left(\frac{E \alpha}{R T_{i}^{2}}\right)^{2}\right)\right.
$$

and assume that $\mathcal{H}_{5}$ is verified then $w<1$, it follows

$$
\begin{gathered}
\left\|T^{n}-T_{h}^{n}\right\|_{0, \Omega}^{2}+\left\|C^{n}-C_{h}^{n}\right\|_{0, \Omega}^{2}+\frac{8}{7(1-w)} k \lambda\left\|\nabla\left(T^{n}-T_{h}^{n}\right)\right\|_{0, \Omega}^{2}+\frac{8}{7(1-w)} k\left(\eta-6(\alpha \rho)^{2}\right)\left\|\nabla\left(C^{n}-C_{h}^{n}\right)\right\|_{0, \Omega}^{2} \\
\lesssim \frac{1}{1-w} k n\left(h^{2 \sigma}+k^{2}\right)+\frac{16 k N_{a}}{7} \frac{1}{1-w}\left(N_{T}\left(\theta_{4}+\frac{1}{2}\right)+N_{C}\left(\theta_{9}+\frac{1}{2}\right)\right) \sum_{i=1}^{n-1}\left\|\psi^{i}-\psi_{h}^{i}\right\|_{1,4}^{2} \\
+\frac{w}{1-w}\left(\sum_{i=1}^{n}\left(\left\|\nabla\left(C^{i}-C_{h}^{i}\right)\right\|_{0, \Omega}^{2}+\left\|\nabla\left(T^{i}-T_{h}^{i}\right)\right\|_{0, \Omega}^{2}\right)\right) .
\end{gathered}
$$

By using the Gronwall lemma with

$$
\begin{gathered}
b_{n}=\left\|T^{n}-T_{h}^{n}\right\|_{0, \Omega}^{2}+\left\|C^{n}-C_{h}^{n}\right\|_{0, \Omega}^{2} \\
a_{n}=\frac{8}{7(1-w)} k \min \left(\lambda, \eta-6(\alpha \rho)^{2}\right)\left(\left\|\nabla\left(T^{n}-T_{h}^{n}\right)\right\|_{0, \Omega}^{2}+\left\|\nabla\left(C^{n}-C_{h}^{n}\right)\right\|_{0, \Omega}^{2}\right)
\end{gathered}
$$

and

$$
\begin{aligned}
c_{n}= & \frac{1}{1-w} k n\left(h^{2 \sigma}+k^{2}\right)+\frac{1}{1-w} \frac{16 k N_{a}}{7}\left(N_{T}\left(\theta_{4}+\frac{1}{2}\right)\right. \\
& \left.+N_{C}\left(\theta_{9}+\frac{1}{2}\right)\right) \sum_{i=1}^{n-1}\left\|\psi^{i}-\psi_{h}^{i}\right\|_{1,4}^{2},
\end{aligned}
$$

we obtain

$$
\begin{aligned}
\left\|T^{n}-T_{h}^{n}\right\|_{0, \Omega}^{2}+\left\|C^{n}-C_{h}^{n}\right\|_{0, \Omega}^{2} & +\frac{8}{7(1-w)} k \min \left(\lambda, \eta-6(\alpha \rho)^{2}\right)\left(\left\|\nabla\left(T^{n}-T_{h}^{n}\right)\right\|_{0, \Omega}^{2}\right. \\
& \left.+\left\|\nabla\left(C^{n}-C_{h}^{n}\right)\right\|_{0, \Omega}^{2}\right) \\
& \lesssim\left(\frac{1}{1-w} k n\left(h^{2 \sigma}+k^{2}\right)+\frac{1}{1-w} \frac{16 k N_{a}}{7}\left(N_{T}\left(\theta_{4}+\frac{1}{2}\right)\right.\right. \\
& \left.\left.+N_{C}\left(\theta_{9}+\frac{1}{2}\right)\right) \sum_{i=1}^{n-1}\left\|\psi^{i}-\psi_{h}^{i}\right\|_{1,4}^{2}\right) \exp \left(\frac{w n}{(1-w) \min \left(\lambda, \eta-6(\alpha \rho)^{2}\right)}\right) .
\end{aligned}
$$

According to the Lemma, 3.1 and 3.2, we can see that

$$
\left|\psi^{n}-\psi_{h}^{n}\right|_{W^{1,4}(\Omega)}^{2} \lesssim k n h^{2 \sigma}+\frac{3 k(\beta g)^{2}}{2 \mu_{p}} \sum_{i=1}^{n}\left\|\left(T^{i}-T_{h}^{i}\right)\right\|_{H^{1}(\Omega)}^{2} .
$$

Then using (3.14) we have

$$
\begin{aligned}
\left\|\psi^{n}-\psi_{h}^{n}\right\|_{W^{1,4}(\Omega)}^{2} \lesssim & k n\left(h^{2 \sigma}+k^{2}\right)+k^{2} n^{2}\left(h^{2 \sigma}+k^{2}\right)+\frac{3(\beta g)^{2}}{2 \mu_{p}} \frac{16 N_{a} k}{7(1-w)}\left(N_{T}\left(\theta_{4}+\frac{1}{2}\right)+N_{C}\left(\theta_{9}+\frac{1}{2}\right)\right) \\
& \times \sum_{j=2}^{n} \sum_{i=1}^{j-1}\left\|\psi^{i}-\psi_{h}^{i}\right\|_{1,4}^{2}
\end{aligned}
$$


We conclude using the discrete Grownel lemma

$$
\left\|\psi^{n}-\psi_{h}^{n}\right\|_{L^{2}\left(0, t, \widetilde{W}^{1,4}(\Omega)\right)}^{2} \lesssim e^{k^{2} n^{2} \frac{3(\beta g)}{2 \mu_{p}} \frac{16 N_{a} k}{7(1-w)}\left(N_{T}\left(\theta_{4}+\frac{1}{2}\right)+N_{C}\left(\theta_{9}+\frac{1}{2}\right)\right)}\left(k n\left(h^{2 \sigma}+k^{2}\right)+k^{2} n^{2}\left(h^{2 \sigma}+k^{2}\right)\right) .
$$

Also, for both of the Temperature, concentration and velocity, we have

Lemma 3.13. We have the following error estimates for the temperature and for the concentration:

$$
\begin{aligned}
& \left\|T^{n}-T_{h}^{n}\right\|_{0, \Omega}^{2}+\frac{8}{7(1-w)} k \min \left(\lambda, \eta-6(\alpha \rho)^{2}\right)\left\|\nabla\left(T^{n}-T_{h}^{n}\right)\right\|_{0, \Omega}^{2} \lesssim\left(h^{2 \sigma}+k^{2}\right)\left(k n+k^{2} n^{2}+k^{3} n^{3}\right), \\
& \left\|C^{n}-C_{h}^{n}\right\|_{0, \Omega}^{2}+\frac{8}{7(1-w)} k \min \left(\lambda, \eta-6(\alpha \rho)^{2}\right)\left\|\nabla\left(C^{n}-C_{h}^{n}\right)\right\|_{0, \Omega}^{2} \lesssim\left(h^{2 \sigma}+k^{2}\right)\left(k n+k^{2} n^{2}+k^{3} n^{3}\right) .
\end{aligned}
$$

and

$$
\left\|\omega^{n}-\omega_{h}^{n}\right\|_{L^{2}(\Omega)}^{2} \lesssim\left(h^{2 \sigma}+k^{2}\right)\left(k n+k^{2} n^{2}+k^{3} n^{3}+k^{4} n^{4}\right) .
$$

Proof. we have

$$
\begin{array}{r}
\left\|T^{n}-T_{h}^{n}\right\|_{0, \Omega}^{2}+\left\|C^{n}-C_{h}^{n}\right\|_{0, \Omega}^{2}+\frac{8}{7(1-w)} k \min \left(\lambda, \eta-6(\alpha \rho)^{2}\right)\left(\left\|\nabla\left(T^{n}-T_{h}^{n}\right)\right\|_{0, \Omega}^{2}+\left\|\nabla\left(C^{n}-C_{h}^{n}\right)\right\|_{0, \Omega}^{2}\right) \\
\lesssim k n\left(h^{2 \sigma}+k^{2}\right)+\frac{16 N_{a} k}{7(1-w)}\left(N_{T}\left(\theta_{4}+\frac{1}{2}\right)+N_{C}\left(\theta_{9}+\frac{1}{2}\right)\right) \sum_{i=1}^{n-1}\left\|\psi^{i}-\psi_{h}^{i}\right\|_{1,4}^{2} .
\end{array}
$$

using the Lemma 3.12, we conclude:

$$
\begin{aligned}
& \left\|C^{n}-C_{h}^{n}\right\|_{0, \Omega}^{2}+\frac{8}{7(1-w)} k \min \left(\lambda, \eta-6(\alpha \rho)^{2}\right)\left\|\nabla\left(C^{n}-C_{h}^{n}\right)\right\|_{0, \Omega}^{2} \lesssim\left(h^{2 \sigma}+k^{2}\right)\left(k n+k^{2} n^{2}+k^{3} n^{3}\right) . \\
& \left\|T^{n}-T_{h}^{n}\right\|_{0, \Omega}^{2}+\frac{8}{7(1-w)} k \min \left(\lambda, \eta-6(\alpha \rho)^{2}\right)\left\|\nabla\left(T^{n}-T_{h}^{n}\right)\right\|_{0, \Omega}^{2} \lesssim\left(h^{2 \sigma}+k^{2}\right)\left(k n+k^{2} n^{2}+k^{3} n^{3}\right),
\end{aligned}
$$

we have also, using the lemma 3.1

$$
\left(1+\frac{k \mu_{p}}{2}\right)\left\|\omega^{n}-\omega_{h}^{n}\right\|_{L^{2}(\Omega)}^{2} \lesssim\left(h^{2 \sigma}+k^{2}\right)\left(k n+k^{2} n^{2}+k^{3} n^{3}+k^{4} n^{4}\right) .
$$

\section{Conclusion}

The fully discretized finite element approximations of reaction front propagation within a porous matrix is studied in this paper. The problem is modelled by a system of equations, coupling the reaction-diffusion equations with the hydrodynamic equations under Darcy law. Due to the incompressibility of the fluid, we have consider the Darcy-Boussinesq approximation. The problem is rewritten using the stream function-vorticity formulation. After choosing the appropriate functional framework for our variational problem, we have proved the existence result for the fully-discrete problem. Furthermore, we have established an optimal a priori estimates on the temperature, the concentration, the stream function and the vorticity.

\section{REFERENCES}

[1] K. Allali, M. Belhaq, K. El Karouni, "Influence of quasi-periodic gravitational modulation on convective instability of reaction fronts in porous media", Comm. Nonl. Sci. Num. Sim., vol. 17, no 4, pp. 1588-1596, 2012.

[2] B.J. Matkowsky, G.I. Sivashinsky, "Propagation of a pulsating reaction front in solid fuel combustion", SIAM Journal on Applied Mathematics, vol. 35, no. 3, pp. 465-478, 1978.

[3] V.A. Volpert, V.A. Volpert, V.M. Ilyashenko, J.A. Pojman, "Frontal polymerization in a porous medium", Chemical engineering science, vol. 53, no. 9, pp. 1655-1665, 1998.

[4] B. Jha, L. Cueto-Felgueroso, R. Juanes, "Quantifying mixing in viscously unstable porous media flows", Physical Review E, vol. 84, no 6, pp. 066312, 2011.

[5] K. Vafai, P.C. Huang, "Analysis of heat transfer regulation and modification employing intermittently emplaced porous cavities", Journal of heat transfer, 116.3, pp. 604-613, 1994. 
[6] D.A. Nield, A. Bejan, Convection in porous media, springer, 2006.

[7] D.A.S. Rees, The stability of Darcy-Bénard convection, Handbook of Porous Media 12, pp. 521-528, 2000.

[8] S. Rionero, "Global non-linear stability in double diffusive convection via hidden symmetries", Int. J. Non-Lin. Mech., vol. 47, no. 1, pp. 61-66, 2012.

[9] O. Maneley, M. Marion, R. Temam, "Equation of combustion in the presence of complex chemistry”, Ind. Univ. Math. J., vol. 42, pp. 941-967, 1993.

[10] A. Agouzal, K. Allali, S. Binna, Mixed Finite Element Approximation of Reaction Front Propagation in Porous Media, International Journal of Engineering and Mathematical Modelling, 2015.

[11] F. Brezzi, M. Fortin, Mixed and Hybrid Finite Element Methods, Springer Verlag, New York, 1991.

[12] P.G. Ciarlet, The Finite Element Method for Elliptic Problems, North-Holland, Amsterdam, New York, Oxford, 1978.

[13] X. Girault, P.A. Raviart, Finite Element Approximation of the Navier-Stokes Equations, Springer-Verlag, Berlin, Heidelberg, New-York, 1986.

[14] G.H. Meyer, Initial Value Methods for Boundary Value Problems, New York, New York: Academic Press, 1973.

[15] C.G. Simader, On Dirichlet's Boundary value Problem: LP- Theory based on Generalization Garding's Inequality, Vol. 268. Springer, 1972.

[16] J.M. Holte, Discrete Gronwall lemma and applications, MAA-NCS meeting at the University of North Dakota, 24 October, 2009. 